hep-th/9701147

January, 1997

\title{
F Theory Vacua in Four Dimensions and Toric Threefolds
}

\author{
KENJI MOHRI \\ National Laboratory for High Energy Physics, Ibaraki 305 Japan \\ mohri@theory.kek.jp
}

\begin{abstract}
We investigate $\mathrm{D}=4, \mathrm{~N}=1 \mathrm{~F}$ theory models realized by type IIB string compactification on toric threefolds. Massless spectra, gauge symmetries, phase transitions associated with divisor contractions and flops, and non-perturbative superpotentials are analyzed using elementary toric methods.
\end{abstract}




\section{Introduction}

F theory 34, 27, 28] provides a remarkable way of $\mathrm{D}=4, \mathrm{~N}=1$ string compactification 110, 9, 22, 32, 25, 35, 36, 23] on a elliptic Calabi-Yau fourfold $\pi: X_{4} \rightarrow B_{3}$. In $\mathrm{F}$ theory, complex moduli and $\mathrm{E}_{8} \times \mathrm{E}_{8}$ gauge bundle moduli of the dual heterotic string are unified into the parameters of $X_{4}$ [28, 8]. F theory also enables us a practical way of evaluating non-perturbative superpotential [36, 15, 9, 23].

The aim of this article is to provide several examples of $\mathrm{D}=4 \mathrm{~F}$ theory models realized by type IIB compactification on toric threefolds [26, 1, 30, 18] and to describe explicitly their massless spectra, gauge symmetries, phase transitions and non-perturbative superpotentials.

The organization of this paper is as follows: In section 2 we describe the elements of $\mathrm{D}=4 \mathrm{~F}$ theory compactification. In section 3 we treat examples of models which don't have non-Abelian gauge symmetry. In particular we describe the quantum numbers and the blowing-up/down transition scheme of the toric Fano threefold models. In section $4 \mathbb{P}^{1}$ bundles over $\mathbb{P}^{2}$ models which we call $\mathbb{G}_{n}$ are analyzed in some detail. Here we compute the physical Hodge numbers as well as the non-Abelian gauge symmetry which arises for generic choices of the parameters. Then we show that the transition between $\mathbb{G}_{n}$ models can be described as a blowing-up/down of toric threefolds. Dual heterotic string description of this transition including non-perturbative vacua is also presented. In section 5 we give a example of the flop transition which interpolate between two heterotic strings compactified on different elliptic Calabi-Yau threefolds. In section 6 we treat $\mathbb{P}^{2}$ bundle over $\mathbb{P}^{1}$ models. In section 7 we deal with $\mathbb{P}^{1}$ bundle over $\mathbb{F}_{a}$ models. Particular emphasis is placed on those models that can be described as Landau-Ginzburg orbifolds. In section 8 we consider the effect of type IIB three-brane wrapped around an exceptional divisor of a toric threefold. We show that it induces a non-perturbative superpotential. We also compute the change in physical Hodge numbers for a contraction of an exceptional divisor. In appendix we show the toric data of some Fano threefolds.

While completing this work we got a preprint [24] via hep-th archive which have some overlap with our results. 


\section{$2 \quad \mathrm{D}=4$ Compactification of $\mathrm{F}$ Theory}

\subsection{Toric Threefold and Elliptic Calabi-Yau Fourfold}

First we describe the construction of a toric threefold $B_{3}$ [26, 37] and the elliptic Calabi-Yau fourfold $X_{4}$ over it [9, 25, 22] which is described as a Weierstrass model [27, 28, 29, 20].

Let $\left(x_{1}, \ldots, x_{n+3}\right)$ be the homogeneous coordinates of $B_{3}, Q_{i}^{a}$ the $a$-th $U(1)$ charge of $x_{i}$, where $a$ runs from 1 to $n$, and

$$
D^{a}:=\sum_{i=1}^{n+3} Q_{i}^{a}\left|x_{i}\right|^{2}-r^{a}
$$

be the $a$-th Hamiltonian. Then the toric threefold $B_{3}$ associated with the above data is defined by the Hamiltonian quotient

$$
B_{3}:=\left\{\left(x_{i}\right) \in \mathbb{C}^{n+3} \mid D^{a}=0\right\} / U(1)^{n} .
$$

If we define the excluded set $E\left(r^{a}\right)$ to be the set of the points the $\mathbb{C}^{* n}$-orbit of which doesn't intersect with $\left\{D^{a}=0, \quad a=1, . ., n\right\}$, then we have another realization of $B_{3}$ as the holomorphic quotient:

$$
B_{3} \cong\left\{\mathbb{C}^{n+3}-E\right\} / \mathbb{C}^{* n}
$$

The total space of radii vectors $\left(r^{a}\right)$ such that $\left\{D^{a}=0, \quad a=1, . ., n\right\}$ is not empty is divided into the Kähler cones according to the topology of $B_{3}$, which constitutes the phase diagram of the model [35].

We realize the elliptic fibration $\pi: X_{4} \rightarrow B_{3}$ as follows:

$$
\left(z_{3}\right)^{2}=\left(z_{2}\right)^{3}+\left(z_{1}\right)^{4} z_{2} F(b)+\left(z_{1}\right)^{6} G(b),
$$

where $\left(z_{1}, z_{2}, z_{3}\right)$ is the homogeneous coordinate of $\mathbb{P}_{(1,2,3)}$ and the coefficients $F$ and $G$ take value in $\Gamma\left(-4 K_{B}\right)$ and $\Gamma\left(-6 K_{B}\right)$ respectively. In terms of toric data, this simply means that $a$-th $U(1)$ charges of $F(x)$ and $G(x)$ are $4 \sum_{i=1}^{n} Q_{i}^{a}$ and $6 \sum_{i=1}^{n} Q_{i}^{a}$ respectively. Note that $X_{4}$ is realized by a hypersurface in the toric fivefold with the homogeneous coordinates $\left(x_{1}, . ., x_{n+3} ; z_{1}, . ., z_{3}\right)$.

In the type IIB picture of $\mathrm{F}$ theory:

$$
\text { F theory on } X_{4}=\text { type IIB theory on } B_{3} \text {, }
$$

the axion/dilaton of type IIB theory $\tau(b):=\phi_{\mathrm{R}}+i \exp \left(-\phi_{\mathrm{NS}}\right)$ depends on the position $b \in B_{3}$ and is identified with the modulus of elliptic fiber over $b$. 
Then the degeneration of elliptic fibers at the discriminant locus

$S_{2}:=\left\{b \in B_{3} \mid 4 F(b)^{3}+27 G(b)^{2}=0\right\}$ is interpreted as the insertion of the seven-brane [21, 19, 34] the world volume of which is $S_{2} \times \mathbb{R}^{4}$.

There is a $D=8$ duality between $\mathrm{F}$ theory and heterotic string:

$$
\text { type IIB string on } \mathbb{P}^{1} \cong \text { heterotic string on } E(\tau) \text {, }
$$

where the axion/dilaton of type IIB side at generic points of $\mathbb{P}^{1}$ is identified with the modulus $\tau$ of the elliptic curve $E$ above [28]. If we consider type IIB compactification on a threefold with a $\mathbb{P}^{1}$ fibration $\pi: B_{3} \rightarrow S_{2}$, then the fiberwise application of (2.5) leads to heterotic string compactified on the elliptic fibered Calabi-Yau threefold $Y_{3}$ over $S_{2}$. If a $\mathbb{P}^{1}$ fibration $B_{3}$ is not a genuine $\mathbb{P}^{1}$ fiber bundle, then the dual heterotic string would be a nonperturbative one with five-branes.

\subsection{Spectrum}

A part of the massless spectrum of $\mathrm{F}$ theory model in four dimensions may be derived from $\mathrm{M}$ theory on $X_{4}$ by further compactifying on $\mathbb{S}^{1}$ owing to the duality:

$$
\text { F theory on } X_{4} \times \mathbb{S}^{1}(R) \cong \mathrm{M} \text { theory on } X_{4} \text {, }
$$

where the area of the fiber torus in the right hand side is $1 / R$. We can then go to the Coulomb phase by giving the VEV to scalars of vector multiplets in $D=3$ [34, 27]. Unfortunately in M theory model above, a vector multiplet is indistinguishable from a chiral multiplet because in three dimensions a vector is equivalent to a scalar.

Thus there would be an ambiguity in identification of the numbers of chiral and vector multiplets. Comparing $M$ theory compactification on $X_{4}$ and type IIB theory compactification on toric $B_{3}$, we propose the following identification of the spectrum

$$
\begin{aligned}
\text { rank (vector multiplets }) & =h^{1,1}\left(X_{4}\right)-\left(h^{1,1}\left(B_{3}\right)+1\right) \\
\#(\text { neutral chiral multiplets }) & =h^{1,1}\left(B_{3}\right)+h^{1,3}\left(X_{4}\right)+h^{1,2}\left(X_{4}\right)
\end{aligned}
$$

\footnotetext{
${ }^{1}$ For any toric threefold, cohomology groups of odd degree vanish. If we take a threefold which is not toric, e.g. $\mathbb{P}^{4}[d], d=2,3,4$, for which $X_{4}$ becomes complete intersection in a toric variety [9], we have generically non-zero $H^{1,2}\left(B_{3}\right)$, which implies that we have rank $h^{1,2}\left(B_{3}\right)$ vector multiplets and $h^{1,2}\left(X_{4}\right)-h^{1,2}\left(B_{3}\right)$ neutral chiral multiplets.
} 
$H^{2,2}$ is also important because they enter into the theory as the vacuum expectation value of the $\mathrm{F} / \mathrm{M} / \mathrm{IIA}$ theory 4 -form field strength on $X_{4}$ [6, 25]. The topological invariants of a Calabi-Yau fourfold are

$$
\begin{aligned}
\chi\left(X_{4}\right) & =4+2\left(h^{1,1}+h^{1,3}\right)+h^{2,2}-4 h^{1,2} \\
\chi\left(T^{*}\right) & =-\left(h^{1,1}+h^{1,3}\right)+h^{1,2}=8-\frac{1}{6} \chi\left(X_{4}\right) \\
\chi\left(\wedge^{2} T^{*}\right) & =h^{2,2}-2 h^{1,2}=12+\frac{2}{3} \chi\left(X_{4}\right) .
\end{aligned}
$$

From the last two equations, we obtain a constraint on the spectrum:

$$
-4\left(h^{1,1}+h^{1,3}\right)+2 h^{1,2}+h^{2,2}=44,
$$

which can be used as a consistency check of the spectra of various models treated below.

\subsection{Spectrum of Toric Hypersurface}

When $X_{4}$ is a hypersurface in a toric fivefold as our case and the Newton polytope $\Delta$ of which is reflexive, the Hodge numbers of a MPCP (maximal projective crepant partial) resolution of $X_{4}$, which is independent of the choice of a MPCP resolution, are determined from the combinatoric data of the ambient toric variety $\left[\begin{array}{|l}4 \\ 5\end{array}\right.$

$$
\begin{aligned}
h^{3,1} & =l(\Delta)-6-\sum_{\operatorname{dim} \Theta=4} l^{0}(\Theta)+\sum_{\operatorname{dim} \Theta=3} l^{0}(\Theta) l^{0}\left(\Theta^{*}\right), \\
h^{2,1} & =\sum_{\operatorname{dim} \Theta=2} l^{0}(\Theta) l^{0}\left(\Theta^{*}\right), \\
h^{1,1} & =l\left(\Delta^{*}\right)-6-\sum_{\operatorname{dim} \Theta=0} l^{0}\left(\Theta^{*}\right)+\sum_{\operatorname{dim} \Theta=1} l^{0}(\Theta) l^{0}\left(\Theta^{*}\right),
\end{aligned}
$$

where $\Theta$ is a face of the Newton polytope $\Delta, l^{0}(\Theta)$ the number of integral points inside $\Theta$, and $\Theta^{*}$ the dual face of $\Theta$ such that $\operatorname{dim} \Theta+\operatorname{dim} \Theta^{*}=4$.

It must be noted here that the reflexivity of the Newton polytope does not assure the existence of a smooth resolution of the Calabi-Yau fourfold $X_{4}$. A MPCP resolution $\hat{X}_{4}$ of $X_{4}$ may leave terminal point singularities that cannot be resolved without destroying the Ricci flat condition.

For example the (non-elliptic) Calabi-Yau fourfold $X_{4}=\mathbb{P}_{(1,1,1,1,2,2)}[8]$ has four $\mathbb{Z}_{2}$ terminal singular points. The physical Hodge numbers are obtained by the Batyrev formula (2.13) or the Vafa formula of Landau-Ginzburg orbifold 
[33]: $\left(h^{1,1}, h^{1,3}, h^{1,2}, h^{2,2}\right)=(1,443,0,1820)$.

As $h^{1,1}=1$, we could say that the physical Hodge numbers are those of the original singular variety $X_{4}$. Compare this with $X_{4}:=\mathbb{P}_{(1,1,1,1,4,4)}[12]$ which has three $\mathbb{Z}_{4}$ singular points which can be blown-up to $\mathbb{P}^{3}$ and $h^{1,1}=1+3$.

We conjecture that string theory on a Calabi-Yau fourfold $X_{4}$ which has at most terminal point singularities is well-defined at the perturbative level as we can realize such models as Landau-Ginzburg or torus orbifolds. Hence we may restrict ourselves here to the Calabi-Yau fourfolds the Newton polytopes of which are reflexive.

Among them the models which can be described as a Landau-Ginzburg orbifold are of importance because (the mirror dual of) the intersection form:

$H^{2,2} \times H^{2,2} \rightarrow \mathbb{C}[6]$, which contributes to the electric charge of $\mathrm{F} / \mathrm{M} / \mathrm{IIA}$ theory $4 / 3 / 2$-form gauge field $C_{(4 / 3 / 2)}$ [6, 17, 25] according to

$$
d \tilde{F}_{(7)}=-\frac{1}{2} F_{(4)} \wedge F_{(4)}+I_{(8)}(R)+\delta^{(8)}(3 / 2 / 1 \text {-Branes }),
$$

can be easily computed from the chiral ring $\mathcal{R}:=\mathbb{C}\left[x_{i}\right] / \partial_{i} W$ except for the absolutely few members that come from twisted sectors. 


\section{Smooth Models}

\subsection{Smooth Elliptic Calabi-Yau Fourfolds}

If the Ricci tensor $R_{i \bar{j}}(b)$ of a threefold $B_{3}$ is positive semi-definite, then type IIB string on $B_{3}$ yields a $\mathrm{N}=1, \mathrm{D}=4$ model which has generically no nonAbelian gauge symmetry, as the elliptic Calabi-Yau fourfold $X_{4}$ over it is smooth for a generic choice of the parameters. These models have a virtue that we can easily compute the Euler number of $X_{4}$,

$$
\frac{1}{24} \chi\left(X_{4}\right)=12+15 c_{1}\left(B_{3}\right) \cdot c_{1}\left(B_{3}\right) \cdot c_{1}\left(B_{3}\right)
$$

and the superpotential anomaly

$$
\chi\left(D_{3}, \mathcal{O}_{D_{3}}\right)=-\frac{1}{2} W_{2} \cdot W_{2} \cdot c_{1}\left(B_{3}\right), \quad W_{2}:=\pi\left(D_{3}\right)
$$

where $D_{3}\left(W_{2}\right)$ is a Euclidean world volume of $\mathrm{F} / \mathrm{M} / \mathrm{IIA}$ theory five-brane (type IIB theory three-brane) which induces a non-perturbative effect [7, 36], and we have used the integration along the elliptic fiber [32.

A threefold is called Fano if its Ricci tensor is positive definite. It serves as a good example of smooth Weierstrass models.

The toric Fano threefolds are completely classified [30] and are given in Figure 2. They are called as $\mathfrak{F}_{n}, 1 \leq n \leq 18$.

\subsection{Phase Structure of $\mathfrak{F}_{12}$}

Here we choose a specific example $\mathfrak{F}_{12}$ to explain blowing-up/down transitions shown in Figure 2.

The toric data of $\mathfrak{F}_{12}$ is given by the following homogeneous co-ordinates and the charge assignments;

$\begin{array}{lllllll} & x_{1} & x_{2} & x_{3} & x_{4} & x_{5} & x_{6} \\ \lambda_{1} & 0 & 1 & 1 & 0 & 0 & 1 \\ \lambda_{2} & 1 & 1 & 1 & 1 & 0 & 0 \\ \lambda_{3} & 0 & 0 & 0 & 1 & 1 & 0 .\end{array}$

The total Kähler cone $\mathcal{K}_{\text {tot }}$ is spanned by the three vectors $\mathcal{K}_{\text {tot }}:=\mathbb{R}_{\geq 0}\left\langle e_{1}, e_{2}, e_{3}\right\rangle$, which is further subdivided into five Kähler cones according to the topology of the resulting toric threefold $\mathfrak{F}_{n}, n=1,2,3,5,12$. The phase diagram is given in Figure 11. The phase transition at each phase boundary is described as follows. 
- On the phase boundary of $\mathrm{V}$ and II, the divisor $\left\{x_{6}=0\right\} \cong \mathbb{P}^{1}\left(x_{2}, x_{3}\right) \times$ $\mathbb{P}^{1}\left(x_{1}, x_{4}\right)$ of $\mathfrak{F}_{12}$ contracts to the two cycle $\mathbb{P}^{1}\left(x_{2}, x_{3}\right)$.

- On the boundary between $\mathrm{V}$ and III, the divisor $\left\{x_{5}=0\right\} \cong \mathbb{P}^{2}\left(x_{1}, x_{2}, x_{3}\right)$ of $\mathfrak{F}_{12}$ contracts to a point.

- On the phase boundary between $\mathrm{V}$ and $\mathrm{IV}$, the $\mathbb{P}^{1}$ fibers of the divisor $\left\{x_{1}=0\right\} \cong \mathbb{F}_{1}\left(x_{2}, x_{3} ; x_{5}, x_{6}\right)$ of $\mathfrak{F}_{12}$ contracts.

- On the phase boundary of III and I, the divisor $\left\{x_{6}=0\right\} \cong \mathbb{P}^{1}\left(x_{1}, x_{4}\right) \times$ $\mathbb{P}^{1}\left(x_{2}, x_{3}\right)$ of $\mathfrak{F}_{5}$ contracts to $\mathbb{P}^{1}\left(x_{1}, x_{4}\right)$.

- On the phase boundary between II and I, the divisor $\left\{x_{5}=0\right\} \cong$ $\mathbb{P}^{2}\left(x_{1}, x_{2}, x_{3}\right)$ of $\mathfrak{F}_{3}$ contracts to a point.

Indeed we expect these phase transitionst to occur by changing the VEV of the chiral supermultiplets associated with the cohomology class $H^{1,1}\left(\mathfrak{F}_{n}\right)$.

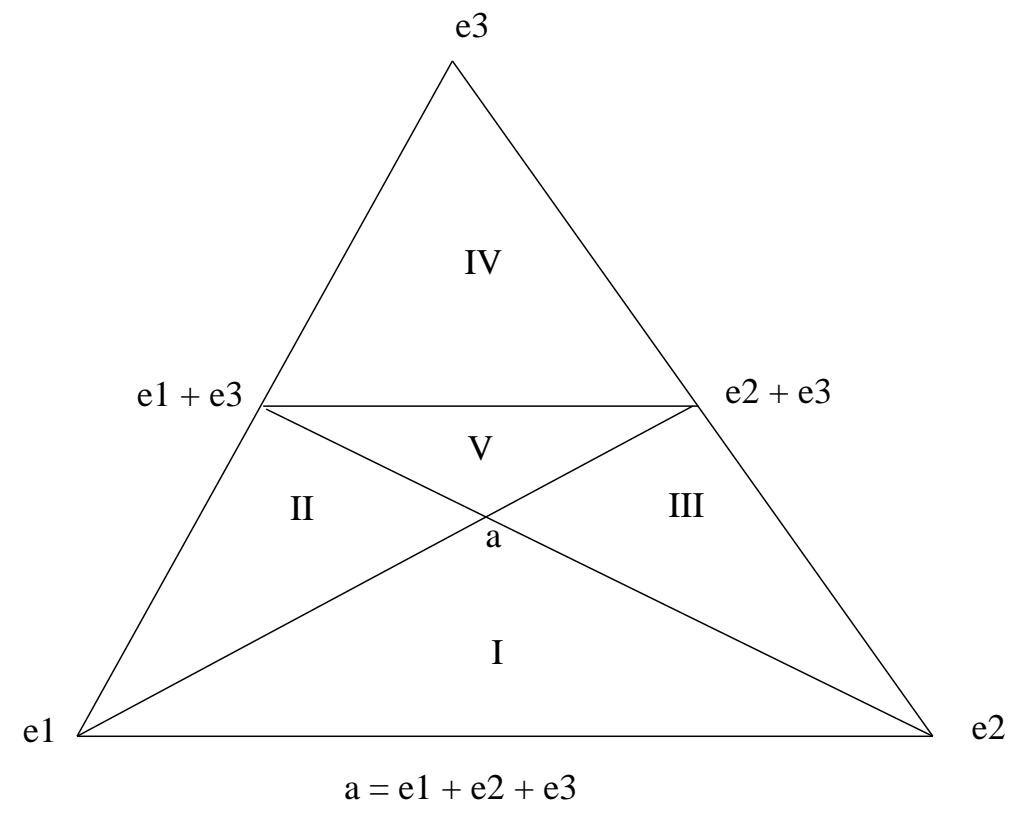

Figure 1: Phase Diagram of $\mathfrak{F}_{12}$ Model

\footnotetext{
${ }^{2}$ Although we don't take into account any quantum corrections to the Kähler moduli space, we expect that pure classical treatment here would suffice to see qualitative structures of the phase transitions.
} 
The phase structure of our model is summarized in Table 1 .

Table 1: Phase Structure of Toric Fano Threefolds

\begin{tabular}{|c|c|c|c|}
\hline & generators of Kähler cone & excluded set $F$ & Fano threefold \\
\hline $\mathrm{I}$ & $\begin{array}{l}e_{1} \\
e_{2} \\
e_{1}+e_{2}+e_{3}\end{array}$ & $\begin{array}{l}\left\{x_{6}=0\right\} \cup \\
\left\{x_{5}=0\right\} \cup \\
\left\{x_{1}=x_{2}=x_{3}=x_{4}=0\right\}\end{array}$ & $\mathfrak{F}_{1}$ \\
\hline II & $\begin{array}{l}e_{1} \\
e_{1}+e_{3} \\
e_{1}+e_{2}+e_{3}\end{array}$ & $\begin{array}{l}\left\{x_{6}=0\right\} \cup \\
\left\{x_{4}=x_{5}=0\right\} \cup \\
\left\{x_{1}=x_{2}=x_{3}=0\right\}\end{array}$ & $\mathfrak{F}_{3}$ \\
\hline III & $\begin{array}{l}e_{2} \\
e_{2}+e_{3} \\
e_{1}+e_{2}+e_{3}\end{array}$ & $\begin{array}{l}\left\{x_{5}=0\right\} \cup \\
\left\{x_{1}=x_{4}=0\right\} \cup \\
\left\{x_{2}=x_{3}=x_{6}=0\right\}\end{array}$ & $\mathfrak{F}_{5}$ \\
\hline IV & $\begin{array}{l}e_{3} \\
e_{1}+e_{3} \\
e_{2}+e_{3}\end{array}$ & $\begin{array}{l}\left\{x_{1}=0\right\} \cup \\
\left\{x_{4}=x_{5}=0\right\} \cup \\
\left\{x_{2}=x_{3}=x_{6}=0\right\}\end{array}$ & $\mathfrak{F}_{2}$ \\
\hline $\mathrm{V}$ & $\begin{array}{l}e_{1}+e_{3} \\
e_{2}+e_{3} \\
e_{1}+e_{2}+e_{3}\end{array}$ & $\begin{array}{l}\left\{x_{1}=x_{4}=0\right\} \cup \\
\left\{x_{5}=x_{6}=0\right\} \cup \\
\left\{x_{1}=x_{2}=x_{3}=0\right\}\end{array}$ & $\mathfrak{F}_{12}$ \\
\hline
\end{tabular}

\subsection{Toric Fano Threefolds}

According to the diagram of the eighteen toric Fano threefolds in Figure 2, there are three members that are on 'the top of the diagram': $\mathfrak{F}_{18}, \mathfrak{F}_{17}$ and $\mathfrak{F}_{11}$. The remaining members are obtained by the successive blowing-downs of one of the above three.

In Figure 2, a solid line with $n(l)$ means a blowing-down of the exceptional divisor $\mathbb{F}_{n}$ with the normal bundle of type $(m, l)=(-1, l)$ (see section 8 ) to $\mathbb{P}^{1}$, while a dotted line means a blowing-down of the exceptional divisor $\mathbb{P}^{2}$ to a point. Therefore it will suffice here to describe only the three top Fano threefolds. The remaining (non-trivial) members shall be found in the subsequent sections and Appendix A. 


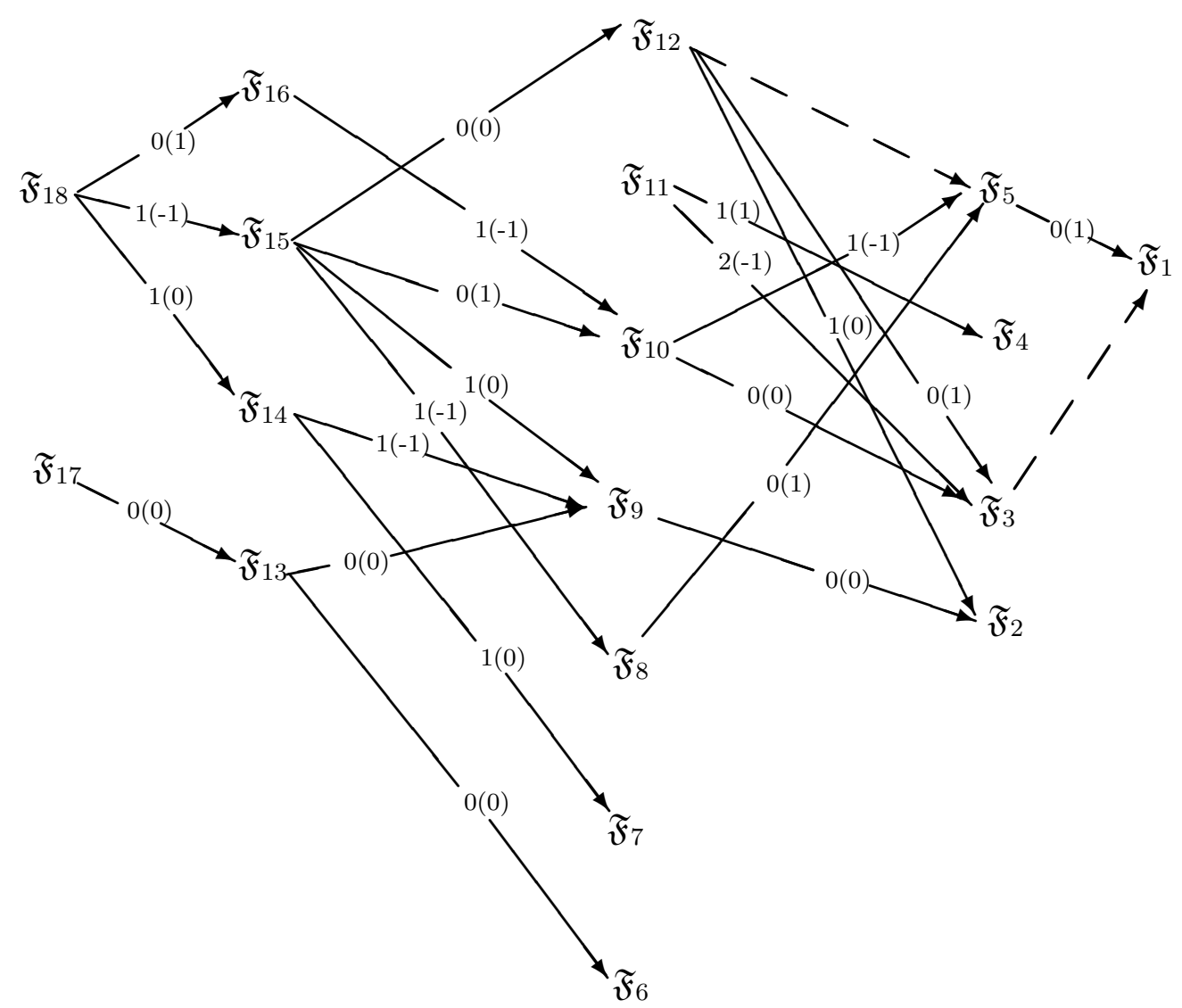

Figure 2: Toric Fano Threefolds 
The toric data of $\mathfrak{F}_{18}$ is given by the following charge assignment.

$\begin{array}{llrrrrrr}x_{7} & x_{8} & x_{1} & x_{3} & x_{5} & x_{2} & x_{4} & x_{6} \\ 1 & 1 & -1 & 0 & 0 & 0 & 0 & 0 \\ 0 & 0 & 1 & 1 & 0 & -1 & 0 & 0 \\ 0 & 0 & 0 & 1 & 1 & 0 & -1 & 0 \\ 0 & 0 & 1 & 0 & 1 & 0 & 0 & -1 \\ 0 & 0 & -1 & -1 & -1 & 1 & 1 & 1 .\end{array}$

We have chosen the combinations of charges so that the excluded set is manifest in this expression, namely the coordinates that has a positive charge, e.g. $\left(x_{7}, x_{8}\right),\left(x_{1}, x_{3}\right),\left(x_{2}, x_{4}, x_{6}\right)$, cannot simultaneously be zero.

It is clear in (3.4) that $\mathfrak{F}_{18}$ has a structure of non-trivial $\mathbb{S}_{3}$ bundle over $\mathbb{P}^{1}$, where $\mathbb{S}_{d}$ is the del Pezzo surface of degree $d$ [28].

The blowing-downs of the divisors $\left\{x_{2,6}=0\right\},\left\{x_{4}=0\right\}$, and $\left\{x_{3,5}=0\right\}$ give rise to $\mathfrak{F}_{15}, \mathfrak{F}_{16}$, and $\mathfrak{F}_{14}$ respectively.

$\mathfrak{F}_{17}$ is isomorphic to $\mathbb{S}_{3} \times \mathbb{P}^{1}$. Its toric data is as follows;

$\begin{array}{llllllll}x_{7} & x_{8} & x_{1} & x_{3} & x_{5} & x_{2} & x_{4} & x_{6} \\ 1 & 1 & 0 & 0 & 0 & 0 & 0 & 0 \\ 0 & 0 & 1 & 1 & 0 & -1 & 0 & 0 \\ 0 & 0 & 0 & 1 & 1 & 0 & -1 & 0 \\ 0 & 0 & 1 & 0 & 1 & 0 & 0 & -1 \\ 0 & 0 & -1 & -1 & -1 & 1 & 1 & 1\end{array}$

Any blowing-down of $\left\{x_{i}=0\right\}, 1 \leq i \leq 6$ leads to $\mathfrak{F}_{13}$.

The toric data of $\mathfrak{F}_{11}$ is as follows.

$\begin{array}{rlllrr}x_{1} & x_{2} & x_{3} & x_{4} & x_{5} & x_{6} \\ 0 & 1 & 1 & 0 & -2 & 1 \\ -1 & 0 & 0 & 1 & 0 & 1 \\ 1 & 0 & 0 & 0 & 1 & -1\end{array}$

There are two exceptional divisors: the blowing-down of $\left\{x_{6}=0\right\}$ and that of $\left\{x_{1}=0\right\}$ give $\mathfrak{F}_{3}$ and $\mathfrak{F}_{4}$ respectively. 


\subsection{Quantum Numbers of Toric Fano Models}

The Euler number of the toric Fano model can be computed by intersection paring formula (3.1). The Hodge numbers of it can also be known by analyzing the combinatoric structure of the Newton polytope $\Delta$ and the polar polytope $\Delta^{*}(2.13)$.

We collect in Table 2 the quantum numbers of the toric Fano threefold models. We also give some identifications of the above models.

Table 2: Quantum Numbers of Toric Fano Models

\begin{tabular}{|l||l|l|l|l|l|}
\hline Model & $h^{1,1}$ & $h^{1,3}$ & $h^{1,2}$ & $h^{2,2}$ & $\chi\left(X_{4}\right) / 24$ \\
\hline $\mathfrak{F}_{1}$ & 2 & 3878 & 0 & 15564 & 972 \\
\hline $\mathfrak{F}_{2}$ & 3 & 3277 & 0 & 13164 & 822 \\
\hline $\mathfrak{F}_{3}$ & 3 & 3397 & 0 & 13644 & 852 \\
\hline $\mathfrak{F}_{4}$ & 3 & 3757 & 0 & 15084 & 942 \\
\hline $\mathfrak{F}_{5}$ & 3 & 3277 & 0 & 13164 & 822 \\
\hline $\mathfrak{F}_{6}$ & 4 & 2916 & 0 & 11724 & 732 \\
\hline $\mathfrak{F}_{7}$ & 4 & 3156 & 0 & 12684 & 792 \\
\hline $\mathfrak{F}_{8}$ & 4 & 2676 & 0 & 10764 & 672 \\
\hline $\mathfrak{F}_{9}$ & 4 & 2916 & 0 & 11724 & 732 \\
\hline $\mathfrak{F}_{10}$ & 4 & 3036 & 0 & 12204 & 762 \\
\hline $\mathfrak{F}_{11}$ & 4 & 3036 & 0 & 12204 & 762 \\
\hline $\mathfrak{F}_{12}$ & 4 & 2796 & 0 & 11244 & 702 \\
\hline $\mathfrak{F}_{13}$ & 5 & 2555 & 0 & 10284 & 642 \\
\hline $\mathfrak{F}_{14}$ & 5 & 2675 & 0 & 11124 & 672 \\
\hline $\mathfrak{F}_{15}$ & 5 & 2435 & 0 & 9804 & 612 \\
\hline $\mathfrak{F}_{16}$ & 5 & 2795 & 0 & 11244 & 702 \\
\hline $\mathfrak{F}_{17}$ & 6 & 2194 & 0 & 8844 & 552 \\
\hline $\mathfrak{F}_{18}$ & 6 & 2194 & 0 & 8844 & 552 \\
\hline
\end{tabular}

$\mathfrak{F}_{1}=\mathbb{P}^{3}, \mathfrak{F}_{2}=\mathbb{P}^{2} \times \mathbb{P}^{1}, \mathfrak{F}_{6}=\mathbb{P}^{1} \times \mathbb{P}^{1} \times \mathbb{P}^{1}, \mathfrak{F}_{9}=\mathbb{F}_{1} \times \mathbb{P}^{1}, \mathfrak{F}_{13}=\mathbb{S}_{2} \times \mathbb{P}^{1}$ 


\subsection{Other Smooth Models}

A Ricci semi-positive toric threefold which is not Fano has a curve $C \cong \mathbb{P}^{1}$, called $(-2)$ curve, about which the type IIB axion-dilaton $\tau$ is constant, i.e. the normal bundle $\mathcal{N}$ of $C$ is a non-compact Calabi-Yau threefold, which has the following expression:

$$
\mathcal{N}=\mathcal{O}_{\mathbb{P}^{1}}(a) \oplus \mathcal{O}_{\mathbb{P}^{1}}(b), \quad a+b=-2 .
$$

This is in contrast to the case of a Fano threefold, where any $\mathbb{P}^{1}$ in it has a normal bundle of the type $(a, b)$ with $a+b \geq-1$, pierced by $10(a+b)+18$ seven-branes [35].

The existence of a $(-2)$ curve $C$ in Ricci semi-positive threefolds is of physical importance because a string with $\mathrm{N}=2$ supersymmetry is produced by wrapping type IIB three-brane around $C$. We give some examples of Ricci semi-positive models and their $(-2)$ curves.

First there are models which has $\mathbb{P}^{1}$ of type $(a, b)=(-1,-1)$. It is well-known that such $\mathbb{P}^{1}$ induces a flop transition. For example, each of the following divisor: $\left\{x_{1}=0\right\}$ of $\mathfrak{F}_{18},\left\{x_{1}=0\right\}$ of $\mathfrak{F}_{14}$, or $\left\{x_{6}=0\right\}$ of $\mathfrak{F}_{7}$ is isomorphic to $\mathbb{F}_{0}$ and can be blown down to give a Ricci semi-positive threefold with a $(-1,-1)$ curve.

For another example we present $\mathcal{T}$ which is obtained by blowing-up $\mathbb{P}^{3}\left(x_{2}, x_{4}, x_{6}, x_{8}\right)$ around the four points $(1,0,0,0),(0,1,0,0),(0,0,1,0)$ and $(0,0,0,1)$ [18. The toric data of $\mathcal{T}$ is as follows.

$\begin{array}{rrrrrrrr}x_{2} & x_{4} & x_{6} & x_{8} & x_{1} & x_{3} & x_{5} & x_{7} \\ -2 & -2 & -2 & -2 & 1 & 1 & 1 & 1 \\ 1 & 1 & 1 & 0 & -1 & 0 & 0 & 0 \\ 1 & 1 & 0 & 1 & 0 & -1 & 0 & 0 \\ 1 & 0 & 1 & 1 & 0 & 0 & -1 & 0 \\ 0 & 1 & 1 & 1 & 0 & 0 & 0 & -1 .\end{array}$

Here we give the quantum numbers of the $\mathcal{T}$ model computed from Batyrev formula:

$$
\left(h^{1,1}, h^{1,3}, h^{1,2}, h^{2,2}\right)=(6,1954,0,7884), \quad \chi\left(X_{4}\right) / 24=492 .
$$

To see the flops that $\mathcal{T}$ admits, it is useful to show the triangulation of $\mathbb{S}^{2}$ defined by the fan of $\mathcal{T}$ [30 in Figure 3 . 


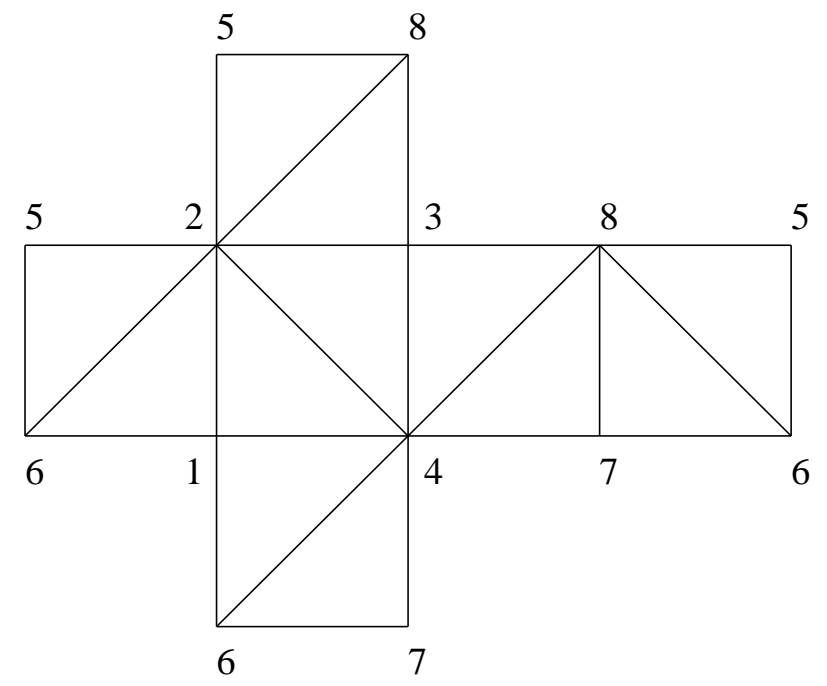

Figure 3: Fan of $\mathcal{T}$

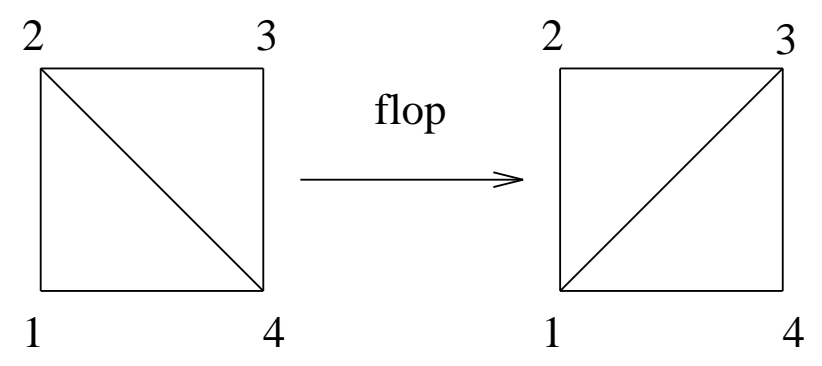

Figure 4: Flop Transition

The flop transition shown in Figure 4 means that $\mathbb{P}^{1}\left(x_{1}, x_{3}\right)$

$\cong\left\{x_{2}=x_{4}=0\right\}$ is contracted and replaced by $\mathbb{P}^{1}\left(x_{2}, x_{4}\right) \cong\left\{x_{1}=x_{3}=0\right\}$.

From the fan in Figure 3 , we see that $\mathcal{T}$ admits six flops;

$$
\begin{array}{lll}
\mathbb{P}^{1}\left(x_{3}, x_{5}\right) \longleftrightarrow \mathbb{P}^{1}\left(x_{2}, x_{8}\right), & \mathbb{P}^{1}\left(x_{1}, x_{5}\right) \longleftrightarrow \mathbb{P}^{1}\left(x_{2}, x_{6}\right), \\
\mathbb{P}^{1}\left(x_{1}, x_{7}\right) \longleftrightarrow \mathbb{P}^{1}\left(x_{4}, x_{6}\right), & \mathbb{P}^{1}\left(x_{1}, x_{3}\right) \longleftrightarrow \mathbb{P}^{1}\left(x_{2}, x_{4}\right), \\
\mathbb{P}^{1}\left(x_{3}, x_{7}\right) \longleftrightarrow \mathbb{P}^{1}\left(x_{4}, x_{8}\right), & \mathbb{P}^{1}\left(x_{5}, x_{7}\right) \longleftrightarrow \mathbb{P}^{1}\left(x_{6}, x_{8}\right) .
\end{array}
$$

Accordingly, $\mathcal{T}$ model gives rise to six tensionless strings carrying $N=2$ supersymmetry. 
Second $\mathbb{O}_{2}$ model is defined by the following toric data:

$\begin{array}{lllll}x_{1} & x_{2} & y_{1} & y_{2} & y_{3} \\ 1 & 1 & 2 & 2 & 0 \\ 0 & 0 & 1 & 1 & 1\end{array}$

where the excluded set is $\left\{x_{1}=x_{2}=0\right\} \cup\left\{y_{1}=y_{2}=y_{3}=0\right\}$.

The curve $\left\{y_{3}=x_{i}=0\right\}$ has the normal bundle $\mathcal{O}_{\mathbb{P}^{1}}(-2) \oplus \mathcal{O}_{\mathbb{P}^{1}}$.

Third $\mathbb{G}_{3}$ model is defined by the toric data:

$\begin{array}{lllll}x_{1} & x_{2} & x_{1} & y_{1} & y_{2} \\ 1 & 1 & 1 & 3 & 0 \\ 0 & 0 & 0 & 1 & 1\end{array}$

where the excluded set is $\left\{x_{1}=x_{2}=x_{3}=0\right\} \cup\left\{y_{1}=y_{2}=0\right\}$.

The curve defined by $\left\{y_{2}=x_{i}=0\right\}$ has the normal bundle $\mathcal{O}_{\mathbb{P}^{1}}(-3) \oplus \mathcal{O}_{\mathbb{P}^{1}}(1)$. 


\section{$4 \quad \mathbb{P}^{1}$ bundle over $\mathbb{P}^{2}$ models}

\subsection{Model}

Here we treat the threefold which we call $\mathbb{G}_{n}$ defined by the toric data below;

$$
\begin{array}{rlllll} 
& x_{1} & x_{2} & x_{3} & y_{1} & y_{2} \\
\lambda & 1 & 1 & 1 & n & 0 \\
\mu & 0 & 0 & 0 & 1 & 1 \\
\mathbb{G}_{n} & :=\left\{\mathbb{C}^{5}-F\right\} / \mathbb{C}_{\lambda}^{*} \times \mathbb{C}_{\mu}^{*},
\end{array}
$$

where the excluded set is $E=\left\{x_{1}=x_{2}=x_{3}=0\right\} \cup\left\{y_{1}=y_{2}=0\right\}$.

It has a $\mathbb{P}^{1}$ fiber bundle structure over $\mathbb{P}^{2}, \pi: \mathbb{G}_{n}(x, y) \rightarrow \mathbb{P}^{2}(x)$. Thus type IIB compactification on $\mathbb{G}_{n}$ is dual to heterotic compactification on the elliptic Calabi-Yau threefold $Y_{3}$ over $\mathbb{P}^{2}$, which can also be realized by the weighted projective hypersurface $\mathbb{P}_{(1,1,1,6,9)}[18]$.

$\mathbb{G}_{n}$ model in $\mathrm{D}=4$ is close analogue of $\mathbb{F}_{n}$ model in $\mathrm{D}=6$ 27, 28. The toric data for the elliptic Calabi-Yau fourfold over $\mathbb{G}_{n}$ is given by

$\begin{array}{cllllllcc} & x_{1} & x_{2} & x_{3} & y_{1} & y_{2} & z_{1} & z_{2} & z_{3} \\ \lambda & 1 & 1 & 1 & n & 0 & 0 & 2(n+3) & 3(n+3) \\ \mu & 0 & 0 & 0 & 1 & 1 & 0 & 4 & 6 \\ \nu & 0 & 0 & 0 & 0 & 0 & 1 & 2 & 3\end{array}$

It can be seen that for $0 \leq n \leq 3, \mathbb{G}_{n}$ model has generically no non-Abelian gauge symmetries. The first three of them are identified with the toric Fano threefolds encountered before; $\mathbb{G}_{0} \cong \mathfrak{F}_{2}, \mathbb{G}_{1} \cong \mathfrak{F}_{3}, \mathbb{G}_{2} \cong \mathfrak{F}_{4}$.

For $4 \leq n$, several seven-branes inevitably coincide on the exceptional cross section divisor $\left\{y_{2}=0\right\}$, producing non-Abelian gauge symmetry.

We have checked using the program PORTA [14] that for $n \geq 3$ the Newton polytope of the elliptic Calabi-Yau fourfold is isomorphic to that of $\mathbb{P}_{(1,1,1, n, 2(n+3), 3(n+3))}[6(n+3)]$, which is reflexive for $n \leq 18$.

Thus we can analyze the spectrum by going to $\mathrm{D}=3 \mathrm{M}$ theory Coulomb phase [27] and then using the Vafa formula for the Landau-Ginzburg orbifold [33 if $\mathbb{P}_{(1,1,1, n, 2(n+3), 3(n+3))}[6(n+3)]$ admits a transverse hypersurface [13, which occurs for $n=3,4,6,9,12,17,18$. We can also apply the Batyrev formula (2.13) even for the remaining cases [11] as in the case of $\mathrm{D}=6$ [12]. 
We list the quantum numbers of the models in Table 3. The singularity

Table 3: Quantum Numbers of $\mathbb{G}_{n}$ Models

\begin{tabular}{|c|c|c|c|c|c|c|}
\hline & $h^{1,1}$ & $h^{1,3}$ & $h^{1,2}$ & $h^{2,2}$ & $\chi\left(X_{4}\right) / 24$ & singularity type \\
\hline$\overline{\mathbb{G}_{\nvdash}}$ & 3 & 3277 & 0 & 13164 & 822 & - \\
\hline $\mathbb{G}_{\nVdash}$ & 3 & 3397 & 0 & 13644 & 852 & - \\
\hline $\mathbb{G}_{\not}$ & 3 & 3757 & 0 & 15084 & $\overline{942}$ & - \\
\hline $\mathbb{G}_{\nVdash}$ & 3 & 4358 & 1 & 17486 & 1092 & - \\
\hline $\mathbb{G}_{q}$ & $\overline{4}$ & 5187 & 0 & 20808 & $1299+3 / 4$ & $I I I$ \\
\hline $\mathbb{G}_{\phi}$ & 5 & 6191 & 0 & 24828 & 1551 & $I_{0}^{* n s}$ \\
\hline $\mathbb{G}_{\phi}$ & 7 & 7341 & 0 & 29436 & 1839 & $I_{0}^{* s}$ \\
\hline $\mathbb{G}_{\varnothing}$ & 7 & 8957 & 0 & 35900 & 2243 & $I V^{* n s}$ \\
\hline $\mathbb{G}_{t}$ & 7 & 10045 & 0 & 40252 & 2515 & $I V^{* n s}$ \\
\hline $\mathbb{G}_{\nrightarrow}$ & 9 & 11587 & 0 & 46428 & 2901 & $I V^{* S}$ \\
\hline $\mathbb{G}_{\nVdash K}$ & 10 & 13255 & 0 & 53104 & $3318+1 / 4$ & $I I I^{*}$ \\
\hline $\mathbb{G}_{\nVdash \nVdash}$ & 10 & 15046 & 0 & 60268 & 3766 & $I I I^{*}$ \\
\hline $\mathbb{G}_{\nVdash Z}$ & 10 & 16959 & 0 & 67920 & $4244+1 / 4$ & $I I I^{*}$ \\
\hline $\mathbb{G}_{\nVdash \nVdash}$ & 12 & 18994 & 6 & 76056 & 4752 & $I I^{*}$ \\
\hline $\mathbb{G}_{\nVdash D}$ & $\overline{12}$ & 21151 & 3 & 84690 & $\overline{5292}$ & $I I^{*}$ \\
\hline $\mathbb{G}_{\nVdash \downarrow}$ & 12 & 23429 & 1 & 93806 & $\overline{5862}$ & $I I^{*}$ \\
\hline $\mathbb{G}_{\nVdash \phi}$ & 12 & 25828 & 0 & 103404 & 6462 & $I I^{*}$ \\
\hline $\mathbb{G}_{\nVdash \varnothing}$ & 12 & 28348 & 0 & 113484 & 7092 & $I I^{*}$ \\
\hline $\mathbb{G}_{\nVdash t}$ & 11 & 30989 & 0 & 124044 & 7752 & $I I^{*}$ \\
\hline
\end{tabular}

type in Table 3 means the one at $\left\{y_{2}=0\right\}$.

As for the non-Abelian gauge symmetry localized at $\left\{y_{2}=0\right\}$, we remark that the prediction (2.7) of the rank of the gauge group $\left(h^{1,1}-3\right)$ seems consistent with the singularity type of the elliptic fibration analyzed by [28, 2, 8]: $\mathrm{SU}_{2}(n=4), \mathrm{G}_{2}(n=5), \mathrm{SO}_{8}(n=6), \mathrm{F}_{4}(n=7,8), \mathrm{E}_{6}(n=9), \mathrm{E}_{7}(n=$ $10,11,12), \mathrm{E}_{8}(n=13-18)$. The reduction of the gauge groups to non-simply laced ones at the singularities of non-split type: $\mathrm{SU}_{2 n} \rightarrow \mathrm{Sp}_{n}, \mathrm{SO}_{8} \rightarrow \mathrm{G}_{2}$, $\mathrm{E}_{6} \rightarrow \mathrm{F}_{4}$ have been explained in [2, 8]. 


\section{$4.2 \mathbb{G}_{n} / \mathbb{G}_{n+1}$ Transition}

If we blow up $\mathbb{G}_{n+1}$ around the toric 2 -cycle $\left\{y_{1}=x_{1}=0\right\} \cong \mathbb{P}^{1}$, we obtain the toric threefold $\mathbb{H}_{n}$ associated with the toric data;

$\begin{array}{cllllcl} & x_{1} & x_{2} & x_{3} & x_{4} & x_{5} & x_{6} \\ \lambda_{1} & 1 & 0 & 1 & 1 & n+1 & 0 \\ \lambda_{2} & 0 & 1 & 1 & 1 & n & 0 \\ \lambda_{3} & 0 & 0 & 0 & 0 & 1 & 1\end{array}$

with the excluded set

$$
E=\left\{x_{1}=x_{5}=0\right\} \cup\left\{x_{2}=x_{6}=0\right\} \cup\left\{x_{1}=x_{3}=x_{4}=0\right\} .
$$

There is an identification; $\mathbb{H}_{1} \cong \mathfrak{F}_{11}$. As $\mathbb{H}_{n}$ can also be obtained by blowing $\mathbb{G}_{n}$ along the curve $\left\{x_{1}=y_{2}=0\right\}$ on the exceptional cross section, we see that $\mathbb{H}_{n}$ model interpolates between two vacua $\mathbb{G}_{n}$ and $\mathbb{G}_{n+1}$ via blowing-up/down of exceptional divisors. The total Kähler cone is generated by $e_{1}, e_{2}, e_{3}$ and subdivided into six Kähler cones as shown in Figure 5.

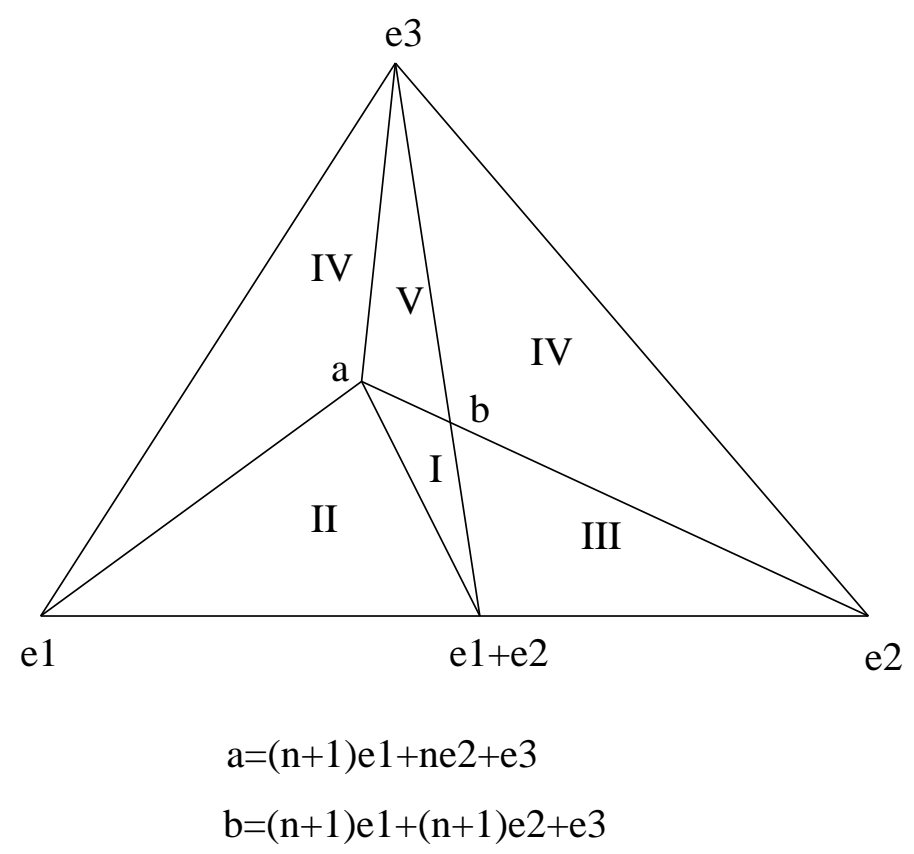

Figure 5: $\quad$ Phase Diagram of $\mathbb{H}_{n}$ Model 
The phase structure is summerized in Table 4 .

Table 4: Phase Structure of $\mathbb{H}_{n}$

\begin{tabular}{|l||l|l|l|}
\hline & generators of Kähler cone & excluded set $E$ & toric threefold \\
\hline \multirow{3}{*}{$\mathrm{I}$} & $e_{1}+e_{2}$, & $\left\{x_{2}=x_{6}=0\right\} \cup$ & \\
& $(n+1) e_{1}+n e_{2}+e_{3}$, & $\left\{x_{1}=x_{5}=0\right\} \cup$ & $\mathbb{H}_{n}$ \\
& $(n+1) e_{1}+(n+1) e_{2}+e_{3}$ & $\left\{x_{1}=x_{3}=x_{4}=0\right\}$ & \\
\hline \multirow{3}{*}{$\mathrm{II}$} & $e_{1}$, & $\left\{x_{1}=0\right\} \cup$ & \\
& $e_{1}+e_{2}$, & $\left\{x_{5}=x_{6}=0\right\} \cup$ & $\mathbb{G}_{n}$ \\
& $(n+1) e_{1}+n e_{2}+e_{3}$ & $\left\{x_{2}=x_{3}=x_{4}=0\right\}$ & \\
\hline \multirow{3}{*}{ III } & $e_{2}$, & $\left\{x_{2}=0\right\} \cup$ & \\
& $e_{1}+e_{2}$, & $\left\{x_{5}=x_{6}=0\right\} \cup$ & $\mathbb{G}_{n+1}$ \\
& $(n+1) e_{1}+(n+1) e_{2}+e_{3}$ & $\left\{x_{1}=x_{3}=x_{4}=0\right\}$ & \\
\hline \multirow{2}{*}{$\mathrm{IV}$} & $e_{1}$, & $\left\{x_{1}=0\right\} \cup$ & \\
& $e_{3}$, & $\left\{x_{6}=0\right\} \cup\left\{x_{2}=x_{3}=\right.$ & $\mathbb{P}_{(1,1,1, n)}$ \\
& $(n+1) e_{1}+n e_{2}+e_{3}$ & $\left.x_{4}=x_{5}=0\right\}$ & \\
\hline \multirow{2}{*}{$\mathrm{V}$} & $e_{3}$, & $\left\{x_{1}=x_{5}=0\right\} \cup$ & \multirow{2}{*}{ singular toric } \\
& $(n+1) e_{1}+n e_{2}+e_{3}$, & $\left\{x_{6}=0\right\} \cup$ & \\
\hline \multirow{2}{*}{$\mathrm{VI}$} & $(n+1) e_{1}+(n+1) e_{2}+e_{3}$ & $\left\{x_{2}=x_{3}=x_{4}=0\right\}$ & \\
& $e_{2}$, & $\left\{x_{2}=0\right\} \cup$ & \\
& $e_{3}$, & $\left\{x_{6}=0\right\} \cup\left\{x_{1}=x_{3}=\right.$ & $\mathbb{P}_{(1,1,1, n+1)}$ \\
\hline
\end{tabular}

The contents of the phase transitions are as follows.

- On the phase boundary between I and II, the divisor $\left\{x_{1}=0\right\}$ $\cong \mathbb{F}_{n+1}\left(x_{3}, x_{4} ; x_{6}, x_{2}\right)$ of $\mathbb{H}_{n}$ contracts to the base $\mathbb{P}^{1}\left(x_{3}, x_{4}\right)$.

- On the phase boundary between I and III, the divisor $\left\{x_{2}=0\right\}$ $\cong \mathbb{F}_{n}\left(x_{3}, x_{4} ; x_{5}, x_{1}\right)$ of $\mathbb{H}_{n}$ contracts to the base $\mathbb{P}^{1}\left(x_{3}, x_{4}\right)$.

- On the phase boundary between I and V, the divisor $\left\{x_{6}=0\right\}$ $\cong \mathbb{P}^{2}\left(x_{1}, x_{3}, x_{4}\right)$ of $\mathbb{H}_{n}$ contracts to a point.

- On the phase boundary between II and IV, the divisor $\left\{x_{6}=0\right\}$ $\cong \mathbb{P}^{2}\left(x_{2}, x_{3}, x_{4}\right)$ of $\mathbb{G}_{n}$ contracts to a point.

- On the phase boundary between III and VI, the divisor $\left\{x_{6}=0\right\}$ $\cong \mathbb{P}^{2}\left(x_{1}, x_{3}, x_{4}\right)$ of $\mathbb{G}_{n+1}$ contracts to a point. 
Recall that the gauge symmetry of $\mathbb{G}_{n}$ model is localized at the world volume of the coincident seven-branes wrapped around the divisor $\left\{y_{2}=0\right\}$. The tension of the resulting three-brane is proportional to the volume of the divisor [28]. Thus at the phase boundary between II and IV (also between III and $\mathrm{VI}$ ), where the volume of the divisor becomes zero, the bare gauge coupling diverses as described in the context of the strong coupling dual of the heterotic string realized by $\mathrm{M}$ theory on $\mathbb{S}^{1} / \mathbb{Z}_{2} \times Y_{3}$ [38, 3].

Let us here describe the dual heterotic coupling and the gauge coupling in terms of the Kähler parameters of $\mathbb{G}_{n}$.

Define the divisors of $\mathbb{G}_{n} d_{1}, d_{2}$ by $\left\{x_{i}=0\right\}=d_{1},\left\{y_{2}=0\right\}=d_{2}$.

Then $\left\{y_{1}=0\right\}$ is identified with $n d_{1}+d_{2}$.

The intersection pairings of them [26] are given by

$d_{1} \cdot d_{1} \cdot d_{1}=0, d_{1} \cdot d_{1} \cdot d_{2}=1, d_{1} \cdot d_{2} \cdot d_{2}=-n, d_{2} \cdot d_{2} \cdot d_{2}=n^{2}$.

The Poincaré dual of the Kähler form is expressed as $\omega=\left(l_{1}+n l_{2}\right) d_{1}+l_{2} d_{2}$, where $l_{2}$ is the radius of fiber $\mathbb{P}_{\text {fiber }}^{1}(y)$ and $l_{1}$ is the radius of the exceptional cross section $\left\{y_{2}=0\right\} \cong \mathbb{P}^{2}(x)$.

Then volumes of toric cycles are evaluated as follows.

$$
\begin{aligned}
\operatorname{Vol}\left(\mathbb{P}_{\text {fiber }}^{1}\right) & =\omega \cdot d_{1} \cdot d_{1}=l_{2} \\
\operatorname{Vol}\left(\left\{y_{2}=0\right\}\right) & =\frac{1}{2 !} \omega \cdot \omega \cdot d_{2}=\frac{1}{2} l_{1}^{2} \\
\operatorname{Vol}\left(\mathbb{G}_{n}\right) & =\frac{1}{3 !} \omega \cdot \omega \cdot \omega=\frac{1}{2}\left(l_{1}^{2}+n l_{1} l_{2}+\frac{1}{3}\left(n l_{2}\right)^{2}\right) l_{2} .
\end{aligned}
$$

We see that the heterotic coupling is given by

$$
\exp (-2 \phi)=\frac{1}{2}\left(l_{1}^{2}+n l_{1} l_{2}+\frac{1}{3}\left(n l_{2}\right)^{2}\right) .
$$

The phase boundary between II and IV is at $l_{1}=0$, where the gauge coupling $g$ $\propto 1 / l_{1}$ diverges [38, 3, 28]. The phase I could be regarded as a non-perturbative heterotic vacuum [16, 31] where a source term of the Bianchi identity for 3form field strength $H_{(3)}$ comes from a five-brane wrapped around a Riemann surface of $Y_{3}$ as pointed out in [38].

Indeed we have two $\mathbb{P}^{1}$ fibration structures in $\mathbb{H}_{n}$ over $\mathbb{P}_{\text {base }}^{2}, \pi_{i}: \mathbb{H}_{n} \rightarrow \mathbb{P}_{\text {base }}^{2}$, $i=1,2$, inherited from those of $\mathbb{G}_{n+1}$ and $\mathbb{G}_{n}$.

The first $\mathbb{P}^{1}$ fibration is defined by

$$
\pi_{1}\left(x_{1}, \ldots, x_{6}\right)=\left(x_{1}, x_{3}, x_{4}\right) .
$$

The generic fiber of $\pi_{1}$ is $\mathbb{P}^{1}$, while the one over the point on the divisor $\left\{x_{1}=0\right\} \subset \mathbb{P}_{\text {base }}^{2}$ is $\mathbb{P}^{1} \cup \mathbb{P}^{1}$. 
The second $\mathbb{P}^{1}$ fibration is defined by

$$
\pi_{2}\left(x_{1}, \ldots, x_{6}\right)=\left(x_{2}, x_{3}, x_{4}\right)
$$

The fiber of $\pi_{2}$ over the divisor $\left\{x_{2}=0\right\} \subset \mathbb{P}_{\text {base }}^{2}$ is $\mathbb{P}^{1} \cup \mathbb{P}^{1}$.

Thus we could say that IIB compactification on a a $\mathbb{P}^{1}$ fiber bundle is dual to a perturbative heterotic string, while one on a $\mathbb{P}^{1}$ fibration which is not a fiber bundle to a non-perturbative heterotic string with five-branes.

In our example, we can see the correspondence between $\mathrm{F}$ theory model and the dual heterotic string model more concretely. The second Chern class of the elliptic Calab-Yau threefold $Y_{3}$ is expressed as

$$
c_{2}\left(T_{Y_{3}}\right)=36\left[\Sigma_{1}\right]+102\left[\Sigma_{2}\right],
$$

where $\left[\Sigma_{1}\right]$ represents the class of a rational curve on the cross section of the elliptic fibration $\pi: Y_{3} \rightarrow \mathbb{P}^{2}$, while $\left[\Sigma_{2}\right]$ the class of a fiber elliptic curve.

Although we don't know the precise way of dividing the second Chern class (4.10) above into those of two $\mathrm{E}_{8}$ gauge bundles in the heterotic string dual to $\mathbb{G}_{n}$ model, we may conjecture that the second Chern classes of two $\mathrm{E}_{8}$ gauge bundles of the corresponding heterotic string have $(18 \pm n)\left[\Sigma_{1}\right]$ as their components and that the heterotic five-brane associated with $\mathbb{H}_{n}$ model is partially wrapped around a Riemann surface $\Sigma_{1}$ on the cross section. It would be interesting to further investigate $(0,2)$ heterotic string compactifications on $Y_{3}$ and elucidate the relation to $\mathrm{F}$ theory models given here. 


\section{$5 \quad$ Flop Transitions}

\subsection{Blowing-Ups of $\mathbb{G}_{n}$ Along a Point}

In this section we present a few examples of the model which admits the flop transition [37, 1] and joins a dual heterotic string vacuum on the elliptic CalabiYau threefold over $\mathbb{F}_{1}$ and that over $\mathbb{P}^{2}$.

First let us take the toric threefolds defined by the data;

$\begin{array}{llllcl}x_{1} & x_{2} & x_{3} & x_{4} & x_{5} & x_{6} \\ 1 & 1 & 1 & 0 & n & 0 \\ 0 & 0 & 1 & 1 & n+1 & 0 \\ 0 & 0 & 0 & 0 & 1 & 1\end{array}$

We show only smooth phases in the phase diagram in Figure 6. The phase I

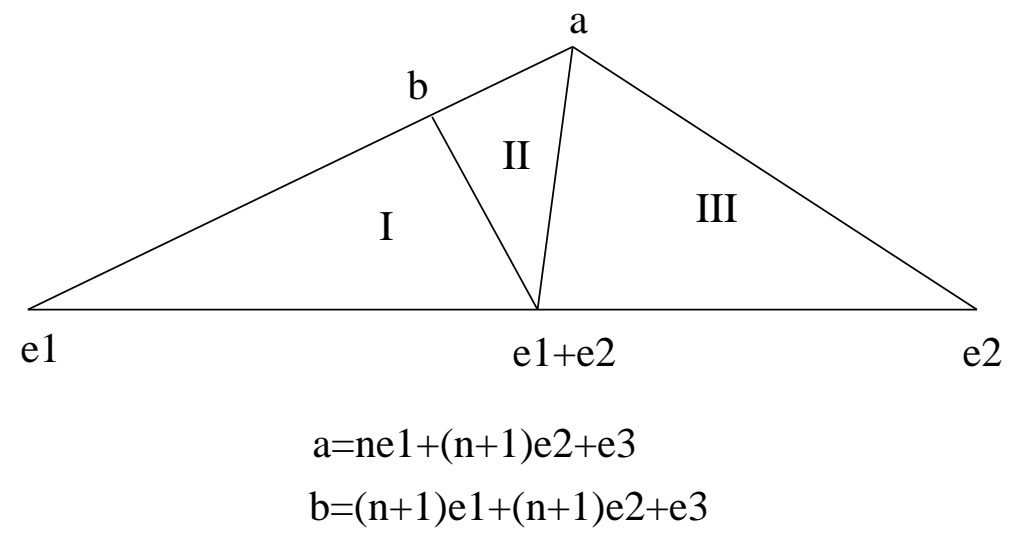

Figure 6: Phase Diagram of Exceptional Blowing-Up of $\mathbb{G}_{n}$

is the $\mathbb{P}^{1}\left(x_{5}, x_{6}\right)$ bundle over $\mathbb{F}_{1}\left(x_{1}, x_{2} ; x_{3}, x_{4}\right)$.

Acrossing the boundary wall between the phase I and II, the 2-cycle

$\mathbb{P}^{1}\left(x_{1}, x_{2}\right) \cong\left\{x_{4}=x_{5}=0\right\}$

of the phase $\mathrm{I}$ is contracted to a point and replaced by the 2-cycle

$\mathbb{P}^{1}\left(x_{4}, x_{5}\right) \cong\left\{x_{1}=x_{2}=0\right\}$

of the phase II. Thus the phase II is a flop of the phase I.

As remarked in [35], there appears the tensionless string carrying $N=2$ supersymmetry at the flop wall. This string is obtained by wrapping the type IIB three-brane around the vanishing 2-cycle above.

On the boundary between the phase II and III, the divisor 
$\mathbb{P}^{2}\left(x_{1}, x_{2}, x_{6}\right) \cong\left\{x_{4}=0\right\}$

of the phase II is blown down to a point. The resulting threefold of the phase III is precisely $\mathbb{G}_{n}$ treated in the previous section. Thus the phase II is nothing but the blowing-up of $\mathbb{G}_{n}$ along the point $\left\{x_{1}=x_{2}=x_{6}=0\right\}$, which is contained in the exceptional cross section.

In the same way, the blowing-up of $\mathbb{G}_{n}$ along the point $\left\{x_{1}=x_{2}=x_{5}=0\right.$ \}, which is not contained in the exceptional cross section, leads to the model with the following toric data.

$\begin{array}{llllcl}x_{1} & x_{2} & x_{3} & x_{4} & x_{5} & x_{6} \\ 1 & 1 & 1 & 0 & n & 0 \\ 0 & 0 & 1 & 1 & n-1 & 0 \\ 0 & 0 & 0 & 0 & 1 & 1\end{array}$

There are three smooth phases as shown in Figure 7 . The phase I is a $\mathbb{P}^{1}$

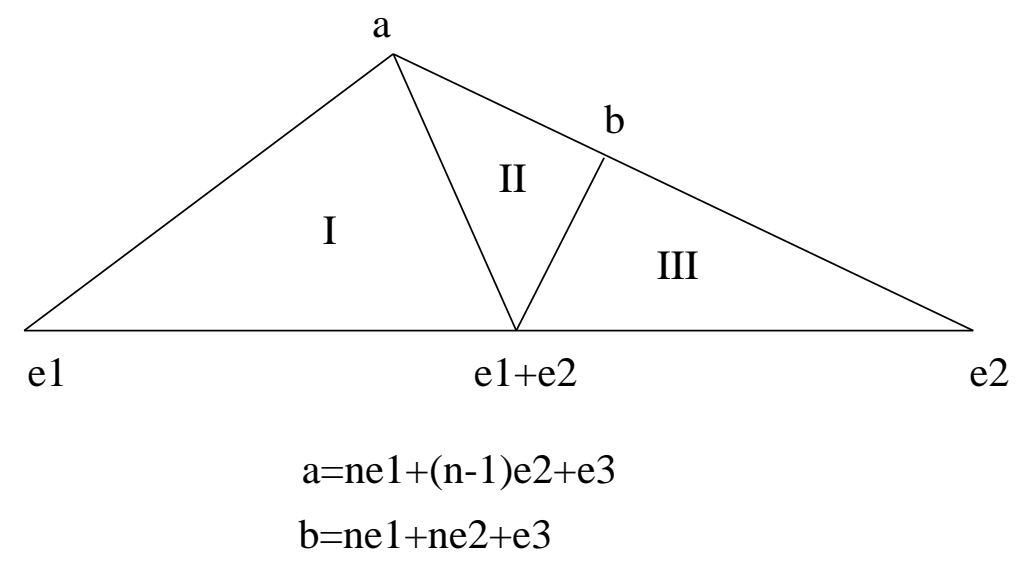

Figure 7: Phase Diagram of Normal Blowing-Up of $\mathbb{G}_{n}$

bundle over $\mathbb{F}_{1}$ and the phase III is $\mathbb{G}_{n}$. The phase boundary between I and II is a flopping wall. hence we have a tensionless string again there.

We have seen in this section that a blowing-up of $\mathbb{G}_{n}$ along a point gives a model which admits a flop transition. Its topology depends on whether the point is in the exceptional cross section or not.

Note that the blowing-up of $\mathbb{G}_{n}$ around a point treated here can be regarded as $\mathbb{P}^{1}$ fibrations over $\mathbb{P}^{2}$, where the fiber over the one point is $\mathbb{P}^{1} \cup \mathbb{P}^{2}$, thus the phase II is also dual to a heterotic string. 


\section{$6 \quad \mathbb{P}^{2}$ bundle over $\mathbb{P}^{1}$ models}

Here we consider the toric threefold defined by the toric data shown below,

$$
\begin{array}{llllll} 
& x_{1} & x_{2} & y_{1} & y_{2} & y_{3} \\
\lambda_{1} & 1 & 1 & n & n & 0 \\
\lambda_{2} & 0 & 0 & 1 & 1 & 1
\end{array}
$$

which we call $\mathbb{O}_{n}$. It has the structure of the $\mathbb{P}^{2}$ fiber bundle over $\mathbb{P}^{1}$ as;

$$
\pi: \mathbb{O}_{n}(x, y) \longrightarrow \mathbb{P}^{1}(x) .
$$

For $0 \leq n \leq 2, \mathbb{O}_{n}$ model has generically completely broken non-Abelian gauge symmetry, while for $2 \leq n \leq 12$ the Newton polytope of the elliptic CalabiYau fourfold over $\mathbb{O}_{n}$ is reflexive and isomorphic to that of

$\mathbb{P}_{(1,1, n, n, 4(n+1), 6(n+1))}[12(n+1)]$, which admits a transverse hypersurface for $n=$

\begin{tabular}{|c|c|c|c|c|c|c|}
\hline & $h^{1,1}$ & $h^{1,3}$ & $h^{1,2}$ & $h^{2,2}$ & $\chi\left(X_{4}\right) / 24$ & singularity type \\
\hline $\mathbb{O}_{\nvdash}$ & 3 & 3277 & 0 & 13164 & 822 & - \\
\hline $\mathbb{O}_{\nVdash}$ & 3 & 3277 & 0 & 13164 & 822 & - \\
\hline $\mathbb{O}_{\not k}$ & 3 & 3277 & 0 & 13164 & 822 & - \\
\hline $\mathbb{O}_{\nVdash}$ & 4 & 3443 & 7 & 13818 & 862 & $I V^{n s}$ \\
\hline $\mathbb{O}_{\not}$ & 5 & 3784 & 13 & 15174 & 946 & $I_{0}^{* n s}$ \\
\hline$\frac{7}{\mathbb{O}_{\phi}}$ & 7 & 4185 & 0 & 16812 & 1050 & $I V^{* n s}$ \\
\hline $\mathbb{O}_{\phi}$ & 7 & 4613 & 12 & 18500 & 1154 & $I V^{* n s}$ \\
\hline $\mathbb{O}_{\not}$ & 10 & 5056 & 0 & 20308 & $1268+2 / 4$ & $I I I^{*}$ \\
\hline $\mathbb{O}_{H}$ & 10 & 5514 & 0 & 22140 & 1383 & $I I I^{*}$ \\
\hline $\mathbb{O}_{\nrightarrow}$ & 12 & 5972 & 24 & 23932 & 1492 & $I I^{*}$ \\
\hline $\mathbb{O}_{\nVdash \nvdash}$ & 12 & 6440 & 12 & 25828 & 1612 & $I I^{*}$ \\
\hline $\mathbb{O}_{\nVdash \nVdash}$ & 12 & 6908 & 0 & 27724 & 1732 & $I I^{*}$ \\
\hline $\mathbb{O}_{\nVdash \not K}$ & 24 & 7376 & 0 & 29644 & 1852 & $I I^{*}$ \\
\hline
\end{tabular}
2,3,4,6,11 and 12 .

We give the quantum numbers of models in Table 5 .

Table 5: Quantum Numbers of $\mathbb{O}_{n}$ Models 
There is an identification: $\mathbb{O}_{1} \cong \mathfrak{F}_{5}$.

The singularity type there means the one at the divisor $\left\{y_{3}=0\right\} \cong \mathbb{P}^{1} \times \mathbb{P}^{1}$. Note that the rank of the vector multiplets $\left(h^{1,1}-3\right)$ predicted in (2.7) doesn't contradict with the following identification of the non-Abelian gauge symmetries localized at $\left\{y_{3}=0\right\}: \mathrm{G}_{2}(n=4), \mathrm{F}_{4}(n=5,6), \mathrm{E}_{7}(n=7,8)$, $\mathrm{E}_{8}(n=9,10,11,12)$, which are obtained from the singularity of the Weierstrass model. There is a remarkable similarity between the singularity of $\mathbb{O}_{n}$ model and that of $\mathrm{D}=6 \mathbb{F}_{n}$ model analyzed in [28]. We also remark that $\mathbb{O}_{n}$ model can be treated as a fiber-wise compactification [22] to $\mathbb{P}_{\text {base }}^{1}$ of $\mathrm{D}=6 \mathbb{P}_{\text {fiber }}^{2}$ model which has the prescribed singularity at $\left\{y_{3}=0\right\}$; namely the parameters of the $\mathrm{D}=6$ fiber theory cannot be chosen arbitrary. 


\section{$7 \quad \mathbb{P}_{1}$ Bundle over $\mathbb{F}_{a}$ Models}

\subsection{Model and Landau-Ginzburg Condition}

Here we consider the threefold $\mathfrak{B}_{a, b, c}$ defined by the following toric data, where $(a, b, c)$ are positive integers,

$\begin{array}{lllllll} & x_{1} & x_{2} & y_{1} & y_{2} & w_{1} & w_{2} \\ \lambda_{1} & 1 & 1 & a & 0 & b & 0 \\ \lambda_{2} & 0 & 0 & 1 & 1 & c & 0 \\ \lambda_{3} & 0 & 0 & 0 & 0 & 1 & 1\end{array}$

with the excluded set $E=\left\{x_{1}=x_{2}=0\right\} \cup\left\{y_{1}=y_{2}=0\right\} \cup\left\{w_{1}=w_{2}=0\right\}$. As $\mathfrak{B}_{a, b, c}$ has a structure of a $\mathbb{P}^{1}(w)$ bundle over $\mathbb{F}_{a}(x, y)$, this model is dual to the heterotic string compactified on the elliptic Calabi-Yau threefold over $\mathbb{F}_{a}$. Alternatively if we take the radius of $\mathbb{P}^{1}(x)$ sufficiently large and regard $\mathfrak{B}_{a, b, c}$ as a $\mathbb{F}_{c}(y, w)$ bundle over $\mathbb{P}^{1}(x)$, this model can be analyzed as a fiberwise compactification of $\mathrm{D}=6$ model on $\mathbb{F}_{c}$ [27, 28] on $\mathbb{P}^{1}$ as proposed in 22].

The toric data for the elliptic Calabi-Yau fourfold over $\mathfrak{B}_{a, b, c}$ realized by the Weierstrass model is as follows,

$\begin{array}{cllllllcc}x_{1} & x_{2} & y_{1} & y_{2} & w_{1} & w_{2} & z_{1} & z_{2} & z_{3} \\ 1 & 1 & a & 0 & b & 0 & 0 & 2(a+b+2) & 3(a+b+2) \\ 0 & 0 & 1 & 1 & c & 0 & 0 & 2(c+2) & 3(c+2) \\ 0 & 0 & 0 & 0 & 1 & 1 & 0 & 4 & 6 \\ 0 & 0 & 0 & 0 & 0 & 0 & 1 & 2 & 3 .\end{array}$

We have observed that the Newton polytope of the $\mathfrak{B}_{a, b, c}$ model is isomorphic to that of $\mathcal{W}_{a, b}:=\mathbb{P}_{(1,1, a, b, 2(a+b+2), 3(a+b+2))}[6(a+b+2)]$ if

$$
\begin{aligned}
a c-b & \geq 0 \\
b-(a+2) & \geq 0 \\
2 b-c(a+2) & \geq 0 .
\end{aligned}
$$

Thus the spectrum of the model satisfying (7.3) can be computed by the Vafa formula of Landau-Ginzburg orbifolds [33] if the corresponding $\mathcal{W}_{a, b}$ admits a transverse hypersurface.

Note that consequently the Newton polytopes of $\mathfrak{B}_{a, b, c}$ model and $\mathfrak{B}_{a, b, c^{\prime}}$ model are isomorphic if both $(a, b, c)$ and $\left(a, b, c^{\prime}\right)$ satisfy the condition (7.3). 
For example let us take the case in which $\mathcal{W}_{a, b}$ admits a Fermat type hypersurface. In this case the facets of the Newton polytope of each $\mathfrak{B}_{a, b, c}$ model with $c$ satisfying (7.3) is simplicial and given by

$$
\begin{aligned}
-m_{1} & \leq 1, \\
-m_{4} & \leq 1, \\
- & m_{5} \leq 1, \\
m_{3}+2\left(2 m_{4}+3 m_{5}\right) & \leq 1, \\
m_{2}-c m_{3}-(c-2)\left(2 m_{4}+3 m_{5}\right) & \leq 1, \\
m_{1}-a m_{2}+(a c-b) m_{3}+(a c-a-b+2)\left(2 m_{4}+3 m_{5}\right) & \leq 1 .
\end{aligned}
$$

Upon the change of the variables over $\mathbb{Z}$ :

$$
\begin{aligned}
m_{i}^{\prime} & =m_{i}, \quad i=1,4,5 \\
-m_{2}^{\prime} & =m_{2}-c m_{3}-2(c-2) m_{4}-3(c-2) m_{5} \\
-m_{3}^{\prime} & =m_{3}+4 m_{4}+6 m_{5},
\end{aligned}
$$

we obtain the well-known Newton polytope of $\mathcal{W}_{a, b}$ :

$$
\begin{aligned}
-m_{1}^{\prime} & \leq 1, \\
-m^{\prime} & \leq 1, \\
-m_{3}^{\prime} & \leq 1, \\
-m^{\prime} & \leq 1, \\
-m^{\prime} & \leq 1, \\
m_{1}^{\prime}+a m^{\prime}{ }_{2}+b m^{\prime}{ }_{3}+(a+b+2)\left(2 m^{\prime}{ }_{4}+3 m^{\prime}\right) & \leq 1 .
\end{aligned}
$$

For another example in favour of our conjecture, take $\mathfrak{B}_{10,48, c}$ models. We have checked that each of $\mathfrak{B}_{10,48,5}, \mathfrak{B}_{10,48,6}, \mathfrak{B}_{10,48,7}$, and $\mathfrak{B}_{10,48,8}$ has a Newton polytope isomorphic to that of $\mathcal{W}_{10,48}$ : the isomorphism between the Newton polytope of $\mathfrak{B}_{10,48, c}$ and that of $\mathcal{W}_{10,48}$ are given by the same form as (77.5). In passing we note that some of $\mathfrak{B}_{a, b, c}$ models (e.g. $\mathfrak{B}_{4,7,2}$ ) have non-reflexive Newton polytopes. It would be interesting if we can classify all the $\mathfrak{B}_{a, b, c}$ models that have reflexive Newton polytopes. 


\subsection{Quantum Numbers}

In this subsection we present the physical Hodge numbers for some models. First we treat $\mathfrak{B}_{3,11,4}$ model. This model has been analyzed as a model of $\mathrm{Sp}_{1}$ gluino condensation using fiberwise compactification technique of dual $\mathrm{D}=6$ heterotic string which has generically an $\mathrm{SO}_{32}$ zero-size instanton [22]

The Newton polytope of the $\mathfrak{B}_{3,11,4}$ model, which is isomorphic to that of $\mathcal{W}_{3,11}$ model, is reflexive with nine facets and eleven vertexes and thus the physical Hodge numbers can be computed by the Batyrev formula (2.13),

$$
\left(h^{1,1}, h^{3,1}, h^{2,1}, h^{2,2}\right)=(10,7497,0,30072) .
$$

Second we list a few examples of the spectra in Table 6 which can be computed by the Vafa formula using the above-mentioned isomorphism of the Newton polytopes.

Table 6: Quantum Numbers of $\mathfrak{B}_{a, b, c}$ Models

\begin{tabular}{|l||l|l|l|l|l|}
\hline$(a, b, c)$ & $h^{1,1}$ & $h^{1,3}$ & $h^{1,2}$ & $h^{2,2}$ & $\chi\left(X_{4}\right) / 24$ \\
\hline$(2,8,2)$ & 8 & 6528 & 0 & 26188 & 822 \\
\hline$(3,10,4)$ & 9 & 6796 & 1 & 27262 & 1703 \\
\hline$(3,15,5)$ & 12 & 10727 & 3 & 42994 & 2686 \\
\hline$(3,27,10)$ & 16 & 24371 & 3 & 97586 & 6098 \\
\hline$(3,30,10)$ & 15 & 28692 & 3 & 114866 & 7178 \\
\hline$(4,36,9)$ & 18 & 30988 & 6 & 124056 & 7752 \\
\hline$(5,14,3)$ & 13 & 7991 & 0 & 32060 & 2003 \\
\hline$(6,16,4)$ & 16 & 8698 & 2 & 34896 & 2180 \\
\hline$(7,11,2)$ & 11 & 6665 & 3 & 26742 & $1670+1 / 4$ \\
\hline$(8,60,8)$ & 30 & 43037 & 3 & 172306 & 10768 \\
\hline$(9,13,2)$ & 12 & 7144 & 0 & 28668 & 1791 \\
\hline$(10,18,3)$ & 18 & 9066 & 4 & 36372 & 2272 \\
\hline$(11,77,7)$ & 43 & 51837 & 0 & 207564 & 12972 \\
\hline$(12,36,3)$ & 32 & 17464 & 0 & 70028 & 4376 \\
\hline
\end{tabular}

We remark that $\mathfrak{B}_{6,16,4}$ model has been treated in 22 as a model of world sheet instanton destabilization, and the resolution of $\mathcal{W}_{2,8}$ model has been given in [10]. 


\section{Superpotentials}

\subsection{Divisor and its Normal Bundle}

In this section we investigate in some detail the generation of superpotential due to type IIB three-brane wrapped around the divisor $W_{2}:=\pi\left(D_{3}\right)$ of $B_{3}$. We shall confine our attention to such divisors that

$$
\pi: D_{3} \longrightarrow W_{2}=\pi\left(D_{3}\right)
$$

which is a divisor of $X_{4}$, is a smooth elliptic threefold.

As the superpotential is generated by wrapping a three-brane around $W_{2}$, we expect that its effect is depend only on the neighbourhood of $W_{2}$ in $B_{3}$ i.e., the normal bundle $\mathcal{N}_{W_{2}}$ of $W_{2}$ in $B_{3}$.

In fact the elliptic threefold $D$ in (8.1) is determined solely by the normal bundle $\mathcal{N}_{W_{2}}$.

Here let us take $\mathbb{G}_{n}$ model for example. It has a distinguished divisor;

$$
W_{2}:=\left\{y_{2}=0\right\} \cong \mathbb{P}^{2}(x),
$$

which is the exceptional cross section.

The normal bundle of this divisor is

$$
\mathcal{N}_{W_{2}} \cong \mathcal{O}_{\mathbb{P}^{2}}(-n)
$$

and it is described as a non-compact toric threefold with the following data:

$$
\begin{array}{lllr}
x_{1} & x_{2} & x_{3} & f \\
1 & 1 & 1 & -n
\end{array}
$$

where $f$ is the fiber coordinate and the excluded set is $\left\{x_{1}=x_{2}=x_{3}=0\right\}$.

Restricting the Weierstrass model over the non-compact threefold $\mathcal{O}_{W_{2}}(-n)$ to the zero section $\{f=0\}$, we arrive at the following toric data for the elliptic threefold $D_{3}$ over $W_{2}$,

$$
\begin{array}{llllcc}
x_{1} & x_{2} & x_{3} & z_{1} & z_{2} & z_{3} \\
1 & 1 & 1 & 0 & 2(3-n) & 3(3-n) \\
0 & 0 & 0 & 1 & 2 & 3
\end{array}
$$

This threefold is smooth only for $n \leq 3$, and we deal with only such cases. The generalization of the above argument to any pair $\left(W_{2}, \mathcal{N}_{W_{2}}\right)$ of the surface 
and its normal bundle is straightforward.

The elliptic threefold $\pi: D_{3} \rightarrow W_{2}$ is described by the following Weierstrass model over $W_{2}$,

$$
\left(z_{3}\right)^{2}=\left(z_{2}\right)^{3}+z_{2}\left(z_{1}\right)^{4} F(w)+\left(z_{1}\right)^{6} G(w),
$$

where $F \in \Gamma\left(-4 K_{W_{2}}+4\left[\mathcal{N}_{\mathcal{W}_{\epsilon}}\right]\right), \quad G \in \Gamma\left(-6 K_{W_{2}}+6\left[\mathcal{N}_{\mathcal{W}_{\epsilon}}\right]\right)$.

Instead of discussing the general theory, it is appropriate here to treat the surface $W_{2}=\mathbb{F}_{n}$, which often appears as a toric divisor.

Let us define the line bundle $\mathcal{O}_{\mathbb{F}_{n}}(l[F]+m[E])$ on $\mathbb{F}_{n}$ by the following toric data,

$$
\begin{array}{lllll}
x_{1} & x_{2} & y_{1} & y_{2} & f \\
1 & 1 & n & 0 & l \\
0 & 0 & 1 & 1 & m
\end{array}
$$

where $[F]$ represents the fiber divisor $\left\{x_{i}=0\right\}$, and $[E]$ represents the exceptional cross section divisor $\left\{y_{2}=0\right\}$ of $\mathbb{F}_{n}$ 30.

If the normal bundle of $W=\mathbb{F}_{n}$ is isomorphic to $\mathcal{O}_{\mathbb{F}_{n}}(l[F]+m[E])$, then the elliptic threefold $\pi: D_{3} \rightarrow W_{2}$ is given by the Weierstrass model over $\mathbb{F}_{n}$ where the charges of $F$ and $G$ with respect to $(8.7)$ are $(4(l+n+2), 4(m+2))$ and $(6(l+n+2), 6(m+2))$ respectively.

We present the toric data of the Weierstrass model in this case for convenience;

$$
\begin{array}{lllllcc}
x_{1} & x_{2} & y_{1} & y_{2} & z_{1} & z_{2} & z_{3} \\
1 & 1 & n & 0 & 0 & 2(n+l+2) & 3(n+l+2) \\
0 & 0 & 1 & 1 & 0 & 2(m+2) & 3(m+2) \\
0 & 0 & 0 & 0 & 1 & 2 & 3
\end{array}
$$

By the investigation of the equation (8.6), we see that the elliptic fibration $\pi: D_{3} \rightarrow W_{2}$ is generically smooth if

$$
(l+n+2)-n(m+2) \geq 0, \text { and } m+2 \geq 0 .
$$

We deal with only such cases. For the case of singular divisors see 23].

A divisor is called exceptional if it can be contracted without leaving any singularities on $B_{3}$. The normal bundle of an exceptional divisor $\mathbb{F}_{n}$ is of type $m=-1$. In fact, under the blowing-down the divisor contracts to $\mathbb{P}^{1}$ as follows,

$$
\left(\mathbb{F}_{n}, \mathcal{O}_{\mathbb{F}_{n}}(l[F]-[E])\right) \longrightarrow\left(\mathbb{P}^{1}, \mathcal{O}_{\mathbb{P}^{1}}(l) \oplus \mathcal{O}_{\mathbb{P}^{1}}(n+l)\right)
$$




\subsection{Detailed Analysis of Superpotential}

Recall that we have presented the superpotential anomaly as an intersection pairing on $B_{3}$ in (3.2). However once we know the normal bundle of $W_{2}$ in $B_{3}$, we can further reduce it to a intersection pairing on the surface $W_{2}$ as follows,

$$
\chi\left(D_{3}, \mathcal{O}_{D_{3}}\right)=-\frac{1}{2}\left[\mathcal{N}_{W_{2}}\right] \cdot\left(c_{1}\left(W_{2}\right)+\left[\mathcal{N}_{W_{2}}\right]\right) .
$$

For the pair $\left(\mathbb{P}^{2}, \mathcal{O}_{\mathbb{P}^{2}}(-n)\right)$, (8.11) gives

$$
\chi\left(D_{3}, \mathcal{O}_{D_{3}}\right)=\frac{1}{2} n(3-n) .
$$

There are the two cases $n=1$ and $n=2$ that satisfy both the necessary condition [36] for the superpotential generation: $\chi\left(D_{3}, \mathcal{O}_{D_{3}}\right)=1$, and the smoothness condition: $n \leq 3$ of the previous subsection.

According to [20], there are the following cohomological relations for any smooth pair $\left(D_{3}, W_{2}\right)$ of an elliptic fibration (8.9);

$$
\begin{aligned}
H^{0}\left(D_{3}, \mathcal{O}_{D_{3}}\right) & \cong H^{0}\left(W_{2}, \mathcal{O}_{W_{2}}\right), \\
0 \rightarrow H^{1}\left(W_{2}, \mathcal{O}_{W_{2}}\right) & \rightarrow H^{1}\left(D_{3}, \mathcal{O}_{D_{3}}\right) \rightarrow H^{2}\left(W_{2}, \mathcal{N}_{W_{2}}\right)^{*} \rightarrow 0, \quad \text { (exact) } \\
0 \rightarrow H^{2}\left(W_{2}, \mathcal{O}_{W_{2}}\right) & \rightarrow H^{2}\left(D_{3}, \mathcal{O}_{D_{3}}\right) \rightarrow H^{1}\left(W_{2}, \mathcal{N}_{W_{2}}\right)^{*} \rightarrow 0, \quad \text { (exact) } \\
H^{3}\left(D_{3}, \mathcal{O}_{D_{3}}\right) & \cong H^{0}\left(W_{2}, \mathcal{N}_{W_{2}}\right)^{*}
\end{aligned}
$$

Using these relations, we can see that both $n=1$ and $n=2$ cases satisfy the sufficient condition for superpotential generation: $h^{i, 0}\left(D_{3}\right)=0, \quad i=1,2,3$. For example, both $\mathbb{G}_{1}$ model and $\mathbb{G}_{2}$ model have a superpotential.

As for the pair $\left(\mathbb{F}_{n}, \mathcal{O}(l[F]+m[E])\right)$, (8.11) yields

$$
\chi\left(D_{3}, \mathcal{O}_{D_{3}}\right)=1-(1+m)(1+l)+\frac{1}{2} m(1+m) n .
$$

The pair $\left(\mathbb{F}_{n}, \mathcal{O}_{\mathbb{F}_{n}}(l[F]+m[E])\right)$ satisfies both the smoothness condition (8.9) and the necessary condition $\chi\left(D_{3}, \mathcal{O}_{D_{3}}\right)=1$ if and only if

$$
m=-1, \quad \text { and } \quad l+2 \geq 0 .
$$

We can show using (8.13) that any divisor which satisfies the condition (8.15) gives rise to the superpotential. Thus all the exceptional divisors $\mathbb{F}_{n}$ appeared in the blowing-up/down transitions of the Fano threefolds in Figure 2 contribute to the superpotential. 


\subsection{Change in Quantum Numbers by Blowing-Down}

By computing the change in intersection pairing $c_{1}(B)^{3}$, we obtain the following formula for the increase of the Euler number under the blowing-down of $\mathbb{F}_{n}$ with the normal bundle of type $(-1, l)$ with $l \geq-2$ :

$$
\frac{1}{24} \Delta \chi=30(3+n+2 l) \text {. }
$$

At least for smooth models this formula should be valid. We may also conjecture that the validity of (8.16) in general as blowing-downs are local operations on threefolds. Then, under the assumption: $\Delta h^{1,1}=-1$, the Hodge numbers of the Calabi-Yau fourfold change as

$$
\begin{aligned}
\Delta h^{1,3} & =1+120(3+n+2 l)+\Delta h^{1,2}, \\
\Delta h^{2,2} & =480(3+n+2 l)+2 \Delta h^{1,2} .
\end{aligned}
$$

Unfortunately $\Delta h^{1,2}$ above is not always zero.

A counter-example is the following: $\mathbb{H}_{2} \rightarrow \mathbb{G}_{2}$, which is associated with the exceptional divisor $\mathbb{F}_{3}$ of type $(-1,-2)$ and has $\Delta h^{1,2}=-1$.

We present for completeness the analogous formula for a blowing-down of exceptional $\mathbb{P}^{2}$ to a point:

$$
\begin{aligned}
\frac{1}{24} \Delta \chi & =30 \cdot 4 \\
\Delta h^{1,3} & =1+120 \cdot 4+\Delta h^{1,2} \\
\Delta h^{2,2} & =480 \cdot 4+2 \Delta h^{1,2} .
\end{aligned}
$$

Indeed the quantum numbers of the toric Fano models $\mathfrak{F}_{n}$ shown in Table 2 are in accord with the formulas (8.16), (8.17) and (8.18) with $\Delta h^{1,2}=0$.

We have also checked that the formula (8.17) with $\Delta h^{1,2}=0$ can be used to the blowing-down: $\mathbb{H}_{n} \rightarrow \mathbb{G}_{n+1}$, where the exceptional divisor is $\mathbb{F}_{n}$ of type $(-1,1)$, which satisfies (8.15). Namely the physical Hodge numbers of the $\mathbb{H}_{n}$ model is known from those of $\mathbb{G}_{n+1}$ model.

\section{Acknowledgement}

The author would like to thank the members of KEK for discussions. 


\section{A Toric data for Fano Threefolds}

We give the toric data of the Fano threefolds. The toric data of $\mathfrak{F}_{14}$ is as follows.

$\begin{array}{lllllrr}x_{7} & x_{8} & x_{2} & x_{4} & x_{6} & x_{5} & x_{1} \\ 1 & 1 & 0 & 0 & 0 & 0 & -1 \\ 0 & 0 & 1 & 0 & 1 & 0 & -1 \\ 0 & 0 & 0 & 1 & 1 & -1 & 0 \\ 0 & 0 & 0 & 0 & -1 & 1 & 1\end{array}$

The toric data of $\mathfrak{F}_{15}$ is as follows.

$\begin{array}{llrlrrr}x_{7} & x_{8} & x_{1} & x_{3} & x_{5} & x_{4} & x_{6} \\ 1 & 1 & -1 & 0 & 0 & 0 & 0 \\ 0 & 0 & 1 & 0 & 1 & 0 & -1 \\ 0 & 0 & 0 & 1 & 1 & -1 & 0 \\ 0 & 0 & 0 & 0 & -1 & 1 & 1\end{array}$

The toric data of $\mathfrak{F}_{16}$ is as follows.

$\begin{array}{llllrrr}x_{7} & x_{8} & x_{3} & x_{5} & x_{1} & x_{6} & x_{2} \\ 1 & 1 & 0 & 0 & -1 & 0 & 0 \\ 0 & 0 & 1 & 0 & 1 & 0 & -1 \\ 0 & 0 & 0 & 1 & 1 & -1 & 0 \\ 0 & 0 & 0 & 0 & -1 & 1 & 1\end{array}$

Each of above three models has a structure of a non-trivial $\mathbb{S}_{2}$ bundle over $\mathbb{P}^{1}$. The toric data of $\mathfrak{F}_{13} \cong \mathbb{P}^{1} \times \mathbb{S}_{2}$ is given by

$\begin{array}{lllllrr}x_{1} & x_{2} & x_{3} & x_{4} & x_{5} & x_{6} & x_{7} \\ 1 & 1 & 0 & 0 & 0 & 0 & 0 \\ 0 & 0 & 0 & 1 & 1 & 0 & -1 \\ 0 & 0 & 1 & 0 & 1 & -1 & 0 \\ 0 & 0 & 0 & 0 & -1 & 1 & 1\end{array}$


The toric data of $\mathfrak{F}_{12}$ model is

$$
\begin{array}{rlllrl}
x_{1} & x_{2} & x_{3} & x_{4} & x_{5} & x_{6} \\
1 & 1 & 1 & 0 & -1 & 0 \\
-1 & 0 & 0 & 0 & 1 & 1 \\
1 & 0 & 0 & 1 & 0 & -1 .
\end{array}
$$

The toric data of $\mathfrak{F}_{8}$ model is

$\begin{array}{llllrr}x_{1} & x_{2} & x_{3} & x_{4} & x_{5} & x_{6} \\ 1 & 1 & 0 & 0 & 0 & -1 \\ 0 & 0 & 1 & 1 & -1 & 0 \\ 0 & 0 & 0 & 0 & 1 & 1\end{array}$

The toric data of $\mathfrak{F}_{7}$ is given by

$\begin{array}{lllllr}x_{1} & x_{2} & x_{3} & x_{4} & x_{5} & x_{6} \\ 1 & 1 & 0 & 0 & 0 & -1 \\ 0 & 0 & 1 & 1 & 0 & -1 \\ 0 & 0 & 0 & 0 & 1 & 1\end{array}$

Both $\mathfrak{F}_{7}$ and $\mathfrak{F}_{8}$ are non-trivial $\mathbb{P}^{1}$ bundles over $\mathbb{P}^{1} \times \mathbb{P}^{1}$.

The toric data of $\mathfrak{F}_{9}$ is given by

$$
\begin{array}{lllrll}
x_{1} & x_{2} & x_{3} & x_{4} & x_{5} & x_{6} \\
1 & 1 & 0 & -1 & 0 & 0 \\
0 & 0 & 1 & 1 & 0 & 0 \\
0 & 0 & 0 & 0 & 1 & 1
\end{array}
$$

so that $\mathfrak{F}_{9}$ is isomorphic to $\mathbb{P}^{1} \times \mathbb{F}_{1}$.

The toric data of $\mathfrak{F}_{10}$ which is a $\mathbb{P}^{1}$ bundle over $\mathbb{F}_{1}$ is given by

$\begin{array}{lllrlr}x_{1} & x_{2} & x_{3} & x_{4} & x_{5} & x_{6} \\ 1 & 1 & 0 & -1 & 0 & 0 \\ 0 & 0 & 1 & 1 & 0 & -1 \\ 0 & 0 & 0 & 0 & 1 & 1\end{array}$




\section{References}

[1] P.S. Aspinwall, G.R. Greene and D.R. Morrison, "Calabi-Yau Moduli Space, Mirror Manifolds and Spacetime Topology Change in String Theory," Nucl. Phys. B416 (1994) 414.

[2] P.S. Aspinwall and M. Gross, "The SO(32) Heterotic String on a K3 Surface," Phys. Lett. B387 (1996) 735.

[3] T. Banks and M. Dine, "Couplings and Scales in Strongly Coupled Heterotic String Theory," hep-th/9605136.

[4] V.V. Batyrev, "Dual Polyhedra and Mirror Symmetry for Calabi-Yau Hypersurfaces in Toric Varieties," J. Alg. Geom. 3 (1994) 493.

[5] V.V. Batyrev and D.I. Dais, "Strong Mckay Correspondence, StringTheoretic Hodge Numbers and Mirror Symmetry," alg-geom/9410001.

[6] K. Becker and M. Becker, "M-Theory on Eight-Manifolds," Nucl. Phys. B477 (1996) 155.

[7] K. Becker, M. Becker and A. Strominger, "Five Branes, Membranes and Non-Perturbative String Theory," Nucl. Phys. B456 (1995) 130.

[8] M. Bershadsky, K. Intriligator, S. Kachru, D.R. Morrison, V. Sadov and C. Vafa, "Geometric Singularities and Enhanced Gauge Symmetries," hep-th/9605200.

[9] I. Brunner, M. Lynker and R. Schimmrigk, "Unification of M- and FTheory Calabi-Yau Four-Fold Vacua," hep-th/9610195.

[10] I. Brunner and R. Schimmrigk, "F Theory on Calabi-Yau Fourfolds," Phys. Lett. B387 (1996) 750.

[11] P. Candelas, X. de la Ossa and S. Katz, "Mirror Symmetry for CalabiYau Hypersurfaces in Weighted $\mathbb{P}_{4}$ and Extension of Landau-Ginzburg Theory," Nucl. Phys. B450 (1995) 267.

[12] P. Candelas and A. Font, "Duality Between the Webs of Heterotic and Type II Vacua," hep-th/9603170,

P. Candelas, E. Perevalov and G. Rajesh, "F-Theory Duals of Nonperturbative Heterotic $E_{8} \times E_{8}$ Vacua in Six Dimensions," hep-th/9606133. 
[13] P. Candelas, M. Lynker and R. Schimmrigk, "Calabi-Yau Manifolds in Weighted $\mathbb{P}_{4}, "$ Nucl. Phys. B341 (1990) 383.

[14] T. Christof, PORTA-A Polyhedron Representation Transformation Algorithm, revised by A. Loebel and M. Stoer, available by anonymous ftp from elib.zib-berlin.de

[15] R. Donagi, A. Grassi and E. Witten, "A Non-Perturbative Superpotential with E-Symmetry," Mod. Phys. Lett. A17 (1996) 2199.

[16] M.J. Duff, R. Minasian and E. Witten, "Evidence for Heterotic/Heterotic Duality," Nucl. Phys. B465 (1996) 413.

[17] S. Ferrara, R. Minasian and A. Sagnotti, "Low-Energy Analysis of $M$ and F Theories on Calabi-Yau Threefolds," Nucl. Phys. B474 (1996) 323.

[18] W. Fulton, Introduction to Toric Varieties, Annals of Mathematical Studies, Number 131, Princeton University Press (1993).

[19] G.W. Gibbons, M.B. Green and M.J. Perry, "Instantons and Seven-Branes in type IIB Superstring Theory," Phys. Lett. B370 (1996) 37.

[20] A. Grassi, "On Minimal Models of Elliptic Threefolds," Math. Ann. 290 (1991) 287.

[21] B.R. Greene, A. Shapere, C. Vafa and S.-T.Yau, "Stringy Cosmic Strings and Noncompact Calabi-Yau Manifolds," Nucl. Phys. B337 (1990) 1.

[22] S. Kachru and E. Silverstein, "Singularities, Gauge Dynamics and Nonperturbative Superpotentials in String Theory," hep-th/9608196.

[23] S. Katz and C. Vafa, "Geometric Engineering of $\mathrm{N}=1$ Quantum Field Theories," hep-th/9611090,

M. Bershadsky, A. Johansen, T. Pantev, V. Sadov and C. Vafa, "F-Theory, Geometric Engineering and N=1 Dualities," hep-th/9612052.

[24] A. Klemm, B. Lian, S.-S. Roan and S.-T. Yau, "Calabi-Yau fourfolds for M- and F- Theory compactifications," hep-th/9701023.

[25] P. Mayr, "Mirror Symmetry, $N=1$ Superpotentials and Tensionless Strings on Calabi-Yau Four-Folds," hep-th/9610162. 
[26] D.R. Morrison and M.R. Plesser, "Summing the Instantons: Quantum Cohomology and Mirror Symmetry in Toric varieties," Nucl. Phys. B450 (1995) 279.

[27] D.R. Morrison and C. Vafa, "Compactifications of F-theory on Calabi-Yau Threefolds - I," Nucl. Phys. B473 (1996) 74.

[28] D.R. Morrison and C. Vafa, "Compactifications of F-theory on Calabi-Yau Threefolds - II," Nucl. Phys. B476 (1996) 437.

[29] N. Nakayama, "On Weierstrass Models," pp.405-431 in Algebraic Geometry and Commutative Algebra in Honor of Masayoshi Nagata, Kinokuniya, Tokyo (1988).

[30] T. Oda, Convex Bodies and Algebraic Geometry: An Introduction to the Theory of Toric Varieties, Ergebnisse der Mathematik und ihrer Grenzgebiete, 3. Folge · Band 15 Springer-Verlag (1988).

[31] N. Seiberg and E. Witten, "Comments on String Dynamics in Six Dimensions," Nucl. Phys. B471 (1996) 121.

[32] S. Sethi, C. Vafa and E. Witten, "Constraints on Low-Dimensional String Compactifications," Nucl. Phys. B480 (1996) 213.

[33] C. Vafa, "Superstring Vacua," pp.145-177 in 1989 Summer School in High Energy Physics and Cosmology, Trieste Italy, The ICTP Series in Theoretical Physics vol.6, J.C. Pati, S. Randjbar-Daemi, E. Sezgin and Q. Shafi eds., World Scientific (1990).

[34] C. Vafa, "Evidence for F-Theory," Nucl. Phys. B469 (1996) 403.

[35] E. Witten, "Phase Transitions in $M$-Theory and F-Theory," Nucl. Phys. B471 (1996) 195.

[36] E. Witten, "Non-Perturbative Superpotentials in String Theory," Nucl. Phys. B474 (1996) 343.

[37] E. Witten, "Phases of $N=2$ Theories in Two Dimensions," Nucl. Phys. B403 (1993) 159.

[38] E. Witten, "Strong Coupling Expansion of Calabi-Yau Compactification," Nucl. Phys. B471 (1996) 135. 